\title{
THIN FILM DEPTH PROFILING BY MeV ION BEAM ANALYSIS
}

Chris Jeynes, University of Surrey Ion Beam Centre, Guildford, England Richard L Thompson, Department of Chemistry, Durham University, England

\section{TABLE OF CONTENTS}

Introduction 2

Examples of obtaining elemental diffusion profiles $\quad 2$

Example of an optical multilayer $\quad 12$

Example of intermixing analysis $\quad 14$

Example of corrosion analysis 16

Example of 3D analysis for geology 19

Conclusions 22

References 23

Problems 25

\section{Figures and Tables}

Table 1. Composition of a clay mineral (see Figure 2a): chemical analysis compared to RBS. 7

Table 2. Solution of antireflection coating (see Figure 9) with Bayesian uncertainties 14

Figure 1. RBS concentration profiles of $\mathrm{Pb}$ in-diffusing into monazites 4

Figure 2a. RBS spectra from a clay mineral with composition given in Table 1.

Figure 2b. RBS spectra from a clay mineral including diffusion profiles.

Figure 3. Diffusion constants in a clay mineral extracted from RBS spectra. 8

Figure 4. RBS of a polymer exposed to alkali treatment in the presence of catalysts. 8

Figure 5. RBS of a photo-sensitive film exposed in-situ to light. $\quad 9$

Figure 6. Thermal silicidation: composite of 250 RBS spectra taken in real time 10

Figure 7. Three spectra from Figure 6 with an artificial neural network solution 11

Figure 8. ANN and conventional solutions from Fig. 6 over the whole annealing range 11

Figure 9. RBS of 21-layer antireflection coating 13

Figure 10 Complex Fe-Si multilayers on quartz as deposited and ion beam mixed. 15

Figure 11. Niépce's 1827 photograph from the collection of the Louvre Museum. 16

Figure 12. IBA data with solution from a corroded region of Niépce's heliograph (see Figure 11) 17

Figure 13. PIXE and complementary techniques for Darwin glass inclusions 19

Figure 14. EBS for various regions of Darwin glass inclusions - see Figure 13. 20

Figure 15. Analysis of a Si-rich area of a Darwin glass (see Figures 13 and 14). 20 


\title{
THIN FILM DEPTH PROFILING BY MeV ION BEAM ANALYSIS
}

\author{
Chris Jeynes, University of Surrey Ion Beam Centre, Guildford, England
}

Richard L Thompson, Department of Chemistry, Durham University, England

\section{Introduction}

This chapter will explore some uses of IBA [1] to determine depth profiles in real materials, mainly using RBS but also involving EBS (including resonant EBS) and PIXE in some examples. Acronyms used in this chapter are defined in Ref [1]. In this chapter the examples are all of analyses which can be carried out using a 1.7 MV Tandem accelerator (for example), so that there is no discussion of MEIS or LEIS examples. We have also deliberately not included any examples of ERD since heavy ion ERD uses (typically) much larger accelerators and completely different instrumentation, and the light ion (helium) ERD frequently carried out on these small accelerators for hydrogen profiling is interpreted in a way very similar to RBS (or EBS).. We also do not discuss analyses carried out on sample cross-sections (as in, for example, Jenneson et al 1998 [2], or Riggs et al [3]) since this is the trivial case of using a sequence of (essentially) bulk analyses to build up a depth profile.

We aim to help the reader to an appreciation of the great power of IBA methods, especially when they are combined (as in the later examples).. The attentive readers will find examples representing a very wide range of problems in materials science, including simple elemental diffusion, reaction kinetics, intermixing of multilayers, and corrosion etc. An interdisciplinary example of materials science and geology is presented in the end to show the real power of the "total IBA" with 3-D mapping capability. Readers will find examples where extraordinarily detailed information can be extracted from quite complicated samples; and they will also occasionally find examples where the solution given by IBA is known to be wrong, pointing to shortcomings in the models (or the databases) used by the IBA codes. We hope that analysts will increasingly publish model analyses in the technical journals, and thereby encourage our community to improve its ability to handle correctly and in increased detail more and more complex and general sample types.

\section{Examples of obtaining elemental diffusion profiles}

Whilst there are many methods available to determine the local motion of molecules in solid materials, ion beam analysis is perhaps uniquely well suited to measure centre of mass diffusion over longer ranges. Characterising long range diffusion is important since it enables us to understand process such as drug release and delivery [4], barrier properties [5] and absorption [6]. 
The best-known example of diffusion is Fickian diffusion in which the flux material across and interface is proportional to the concentration gradient. By considering the flux of material in and out of an infinitesimal slice, the rate of change of composition within that slice is given by the second derivative of the concentration with respect to distance. The constant of proportionality, $D$, is diffusion coefficient defined by Fick's second law:

$\frac{\partial c}{\partial t}=D \frac{\partial^{2} c}{\partial x^{2}}$

where $c$ is concentration, $t$ is time and $x$ is depth. Depending on the boundary conditions and the response of the material to the diffusing species, various solutions to equation 1 are possible. When the penetrating species comes from a relatively mobile bulk phase, such as a liquid or vapour in contact with a solid host material, diffusion into the solid has no effect on the diffusant concentration at the surface. If the diffusion coefficient in the host material is independent of the concentration, then $\mathrm{D}$ is given by

$c(x, t)=c(0,0) \operatorname{erfc}\left(\frac{x}{2 \sqrt{D t}}\right)$

This method was used by Cherniak et al [7] to characterise the diffusion coefficient of $\mathrm{Pb}$ into $\mathrm{CePO}_{4}$ monazites (see Figure 1). Good agreement between the experimental profiles and Fickian diffusion was confirmed by inverting the data through the error function, i.e. a plot of $\operatorname{erf}^{-1}\left(\frac{c(0,0)-c(x, t)}{c(0,0)}\right)$ versus $x$ does in this case successfully yield a straight line plot of slope $\frac{1}{2 \sqrt{D t}}$.

In reality the diffusion process are often more complex. The diffusing species may have a profound effect on the properties of the material into which it is diffusing and consequently the process cannot be described by a single simple diffusion coefficient. The ability of ion beam analysis to capture an entire concentration profile in a single measurement makes these techniques ideal for studying one dimensional diffusion problems. When diffusion occurs over ranges that exceed the conveniently accessible range of ion beams (a few microns) it is equally possible to quantify diffusion by scanning a micro-focussed ion beam across the fracture surface [3]. Any material provided it can be frozen or vitrified for the duration of an experiment can be studied in this way. Kramer et al [5] pioneered the 


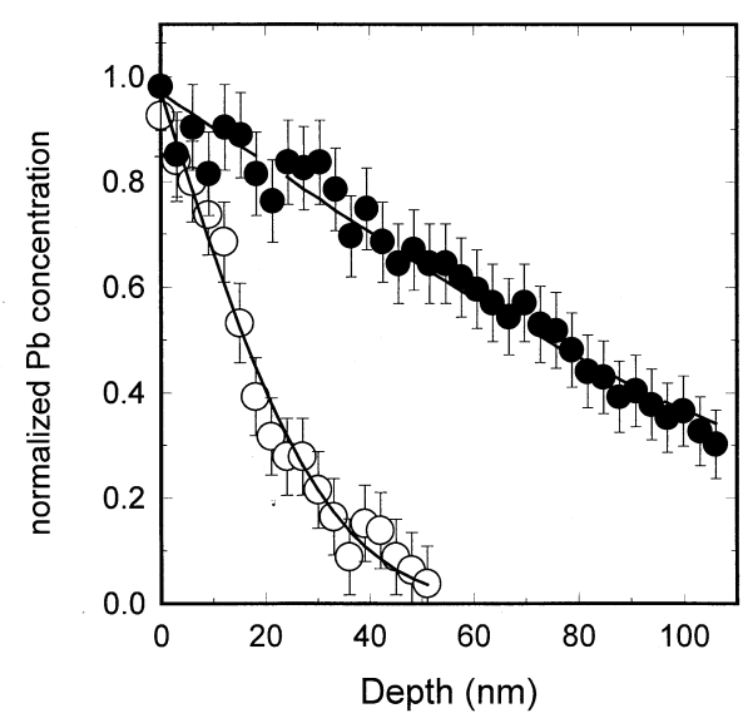

Figure 1. RBS concentration profiles of $\mathrm{Pb}$ in-diffusing into a synthetic monazite (closed circles) and natural monazite (open circles), with fitted erfc function Curves (lines). Reproduced from Figure 1a of Cherniak et al, Geochim Cosmochim Acta 68, 2004, 829-840 (ref [6]).

use of ion beam analysis to study diffusion of liquids into glassy polymers using liquid nitrogen to trap the diffusing species for the duration of the measurements. In this kind of experiment, the chemistry of the diffusants is usually well-defined, for example a series of iodoalkanes, so this information can be applied to infer molecular diffusion via the backscattering from just the constituent iodine atoms. These were key experiments in testing the case II diffusion theory put forward by Thomas and Windle some years earlier [8]. Prior to ion beam analysis experiments this model is based on observations of mass change with time but it was not possible to observe the initial concentration profile of the penetrant.

When diffusion occurs from a thin surface layer into the host material the total volume of the diffusing species must be independent of time and the concentration profile is given by

$c(x, t)=\frac{c(0,0)}{2}\left[\operatorname{erf}\left(\frac{h-x}{2 \sqrt{D t}}\right)+\operatorname{erf}\left(\frac{h+x}{2 \sqrt{D t}}\right)\right]$

where $h$ is the thickness of the surface layer. This functional form has been widely used to study rates and mechanisms of diffusion in polymers. There is in fact frequently sufficient detail within the ion beam analysis data to reveal that this functional form is not completely appropriate and therefore ion beam analysis has successfully been employed to challenge and improve our understanding of diffusion processes [9]. 
Rates of diffusion are acutely sensitive to the thermodynamics of mixing but very hard to predict $a$ priori without quantitative experimental data. The term "thermodynamic slowing" has been used to describe the dramatic decrease in diffusion coefficient that may result from small positive enthalpy of mixing between two species [10,11]. This problem applies particularly interdiffusion where the concentration of the diffusant remains significant even after diffusion has commenced. If the layer of the diffusing species is initially extremely small then it effectively becomes diluted as soon as diffusion begins, and under these circumstances the tracer diffusion this component is independent of the thermodynamics of mixing. Deuterium labelling has commonly been applied to determine the diffusion of one organic material into another [4,10-12]. Here, some or all of the hydrogen atoms of one component in the mixture are substituted for deuterium, which has minimal impact on the chemical and physical properties, but enables direct determination by elastic recoil detection and nuclear reaction analysis. However, even this most innocuous of labelling systems can subtly change the thermodynamics of mixing in a manner that can be detected by ion beam analysis studies of diffusion coefficients. One rather ingenious solution to this problem was to selectively label the diffusing component with bromine only after diffusion was complete [13]. However under such circumstances, care must be taken to account for the impact of the label on the stopping power of the material.

What is perhaps most remarkable about ion beam analysis study diffusion is that it applies not only to simple systems but equally to arbitrarily complex materials. Our ability to label just a single component within any material makes this technique perfect for studying anything from nano composites to biomaterials.

Moncoffre et al (1998) [14] from the Lyons group have made an interesting review of Fickian diffusion in various types of materials (including calculational details, which is why it is mentioned), including (i) obtaining (by $1.5 \mathrm{MeV} \mathrm{He-RBS)} \mathrm{the} \mathrm{diffusion} \mathrm{coefficients} \mathrm{of} \mathrm{La} \mathrm{diffusing} \mathrm{in}$ hydroxyapatite; (ii) obtaining (by proton-NRP using ${ }^{15} \mathrm{~N}(\mathrm{p}, \alpha \gamma){ }^{12} \mathrm{C}$ near the $429 \mathrm{keV}$ resonance) the coefficients of nitrogen diffusion in aluminium; and (iii) obtaining (by $2.5 \mathrm{MeV} \mathrm{He-ERD} \mathrm{with} \mathrm{an} E \mathbf{X}$ $B$ filter instead of an absorber foil for the primary scattered beam) the coefficients of $H$ diffusion in $\mathrm{SiC}: \mathrm{H}$ under D-implantation. The first example is relevant to the immobilisation of radioactive waste, the second to the growth properties of an important refractory material aluminum nitride (AlN), and the third to investigating fusion vessel (tokomak) wall materials.

Alonso et al [15] are also interested in the problem of nuclear waste repositories, this time from the geological point of view. Measurement of diffusion coefficients by "classical" methods usually involves waiting for the diffusion to give measurable concentrations over length scales of cms. But this would involve unfeasibly long experiments for the geological timescales appropriate to these 
applications. Therefore, they turned to nuclear methods (RBS) to measure diffusion in clays over submicron length scales. This paper is very explicit (helpfully to us!) and we will consider it in some detail. Figure 2a shows the RBS spectrum from the clay in Alonso et al's study, and Table 1 shows the analysis they made of this spectrum. This table is an example of over-interpretation of data - see Problem 1. Figure $2 \mathrm{~b}$ shows one of the diffusion profiles studied by these authors, and Figure 3 shows the error function profiles fitted to the RBS data. The diffusion equation constants are obtained directly from these fits (see Table 5 of the paper) since the code used to interpret this data supports fitting with $\boldsymbol{e r f} \boldsymbol{c}$ functions. These data are considered more closely in Problem 1.

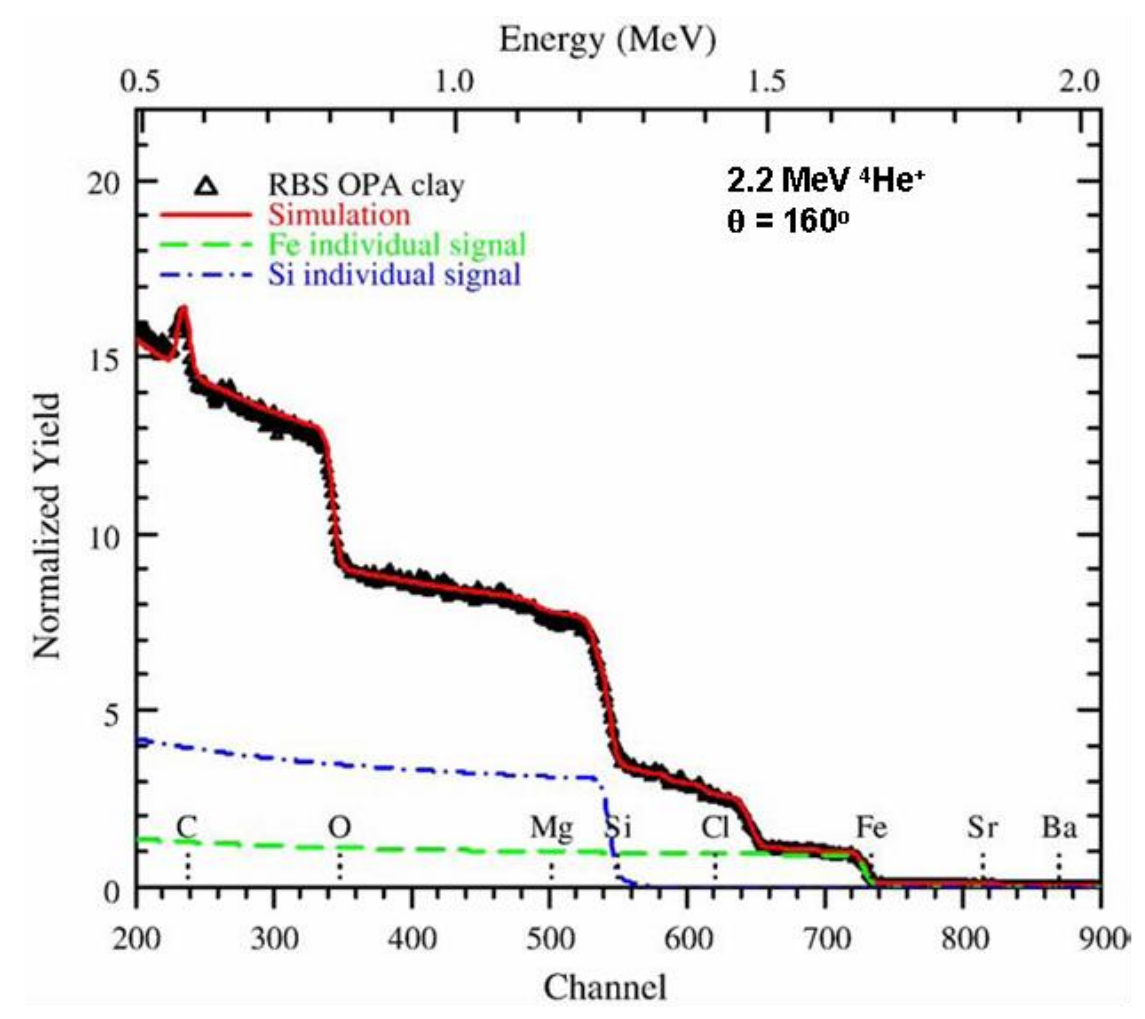

Figure 2a. RBS spectrum from a clay mineral with composition given in Table 1.

Reproduced from Figure 2 of Alonso et al, Applied Clay Science 43, 2009, 477-484 (ref [15]). 


\section{Table 4}

Comparison of the mean elemental compositions obtained for the OPA clay: from chemical analyses (Fernández, 2007) and from the simulated RBS spectra

\begin{tabular}{lll}
\hline Element & \multicolumn{2}{l}{ Composition in atomic mass \% } \\
\cline { 2 - 3 } & (Fernández, 2007) & RBS \\
\hline $\mathrm{C}$ & 4.6 & 4.4 \\
$\mathrm{O}$ & 67 & 65 \\
$\mathrm{Na}$ & $5.2 \cdot 10^{-1}$ & $6.1 \cdot 10^{-1}$ \\
$\mathrm{Mg}$ & 1.1 & 2.1 \\
$\mathrm{Al}$ & 10 & 7.9 \\
$\mathrm{Si}$ & 10 & 16 \\
$\mathrm{~S}$ & $1.6 \cdot 10^{-1}$ & $1.0 \cdot 10^{-1}$ \\
$\mathrm{Cl}$ & $1.4 \cdot 10^{-2}$ & $1.4 \cdot 10^{-2}$ \\
$\mathrm{~K}$ & 1.8 & 2.2 \\
$\mathrm{Ca}$ & 1.2 & 1.7 \\
$\mathrm{Ti}$ & $3.1 \cdot 10^{-1}$ & $1.2 \cdot 10^{-1}$ \\
$\mathrm{Mn}$ & $2.9 \cdot 10^{-2}$ & $1.2 \cdot 10^{-2}$ \\
$\mathrm{Fe}$ & 2.6 & 1.4 \\
$\mathrm{Sr}$ & $6.0 \cdot 10^{-3}$ & $1.6 \cdot 10^{-2}$ \\
$\mathrm{Ba}$ & $4.0 \cdot 10^{-3}$ & $1.8 \cdot 10^{-2}$ \\
\hline
\end{tabular}

Values presented in atomic mass \%.

Table 1. (Table 4 of Alonso et al 2009.) Composition of a clay mineral: chemical analysis compared to RBS.

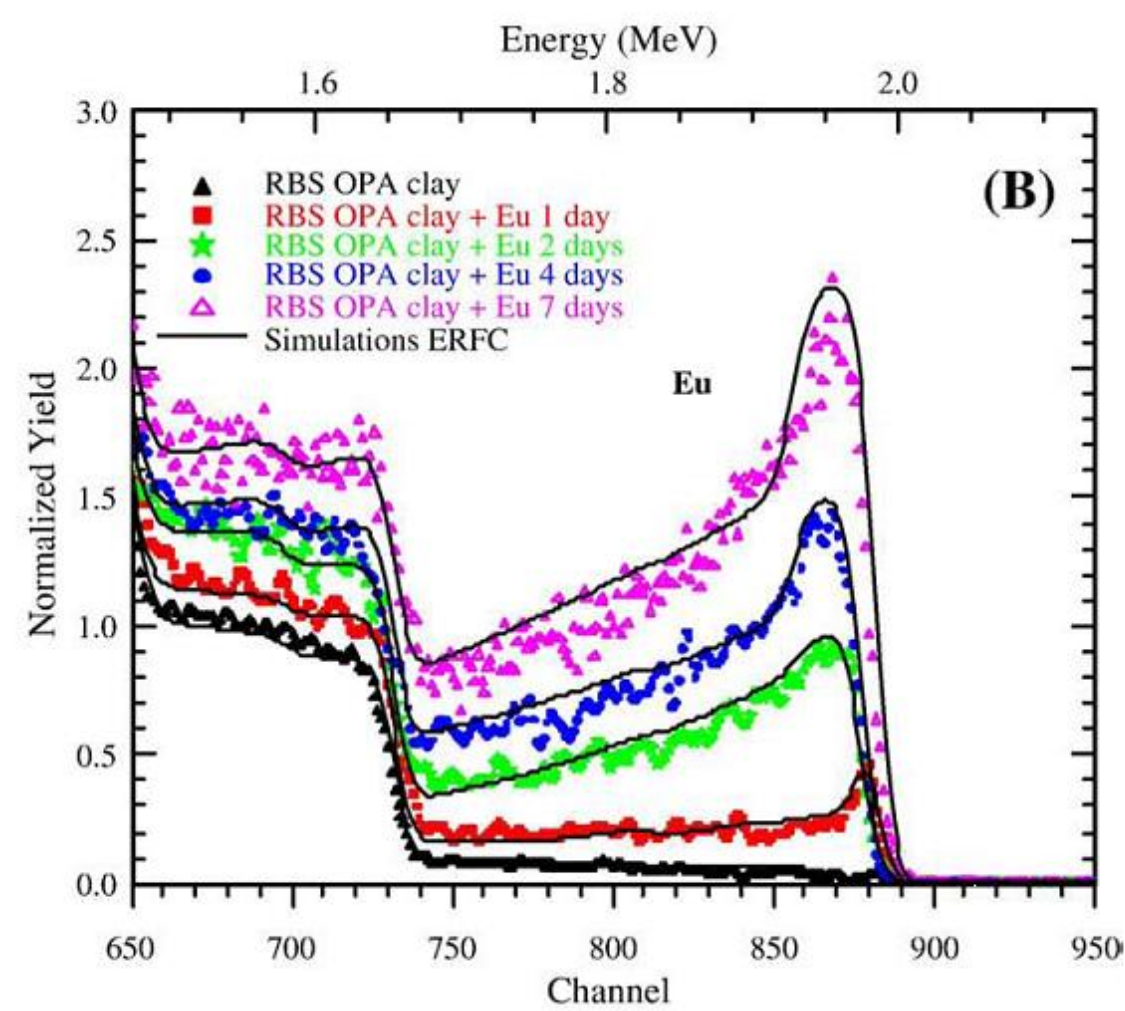

Figure $2 \mathrm{~b}$. RBS spectra from a clay mineral including diffusion profiles. Reproduced from Figure 4b of Alonso et al, Applied Clay Science 43, 2009, 477-484 (ref [15]). 


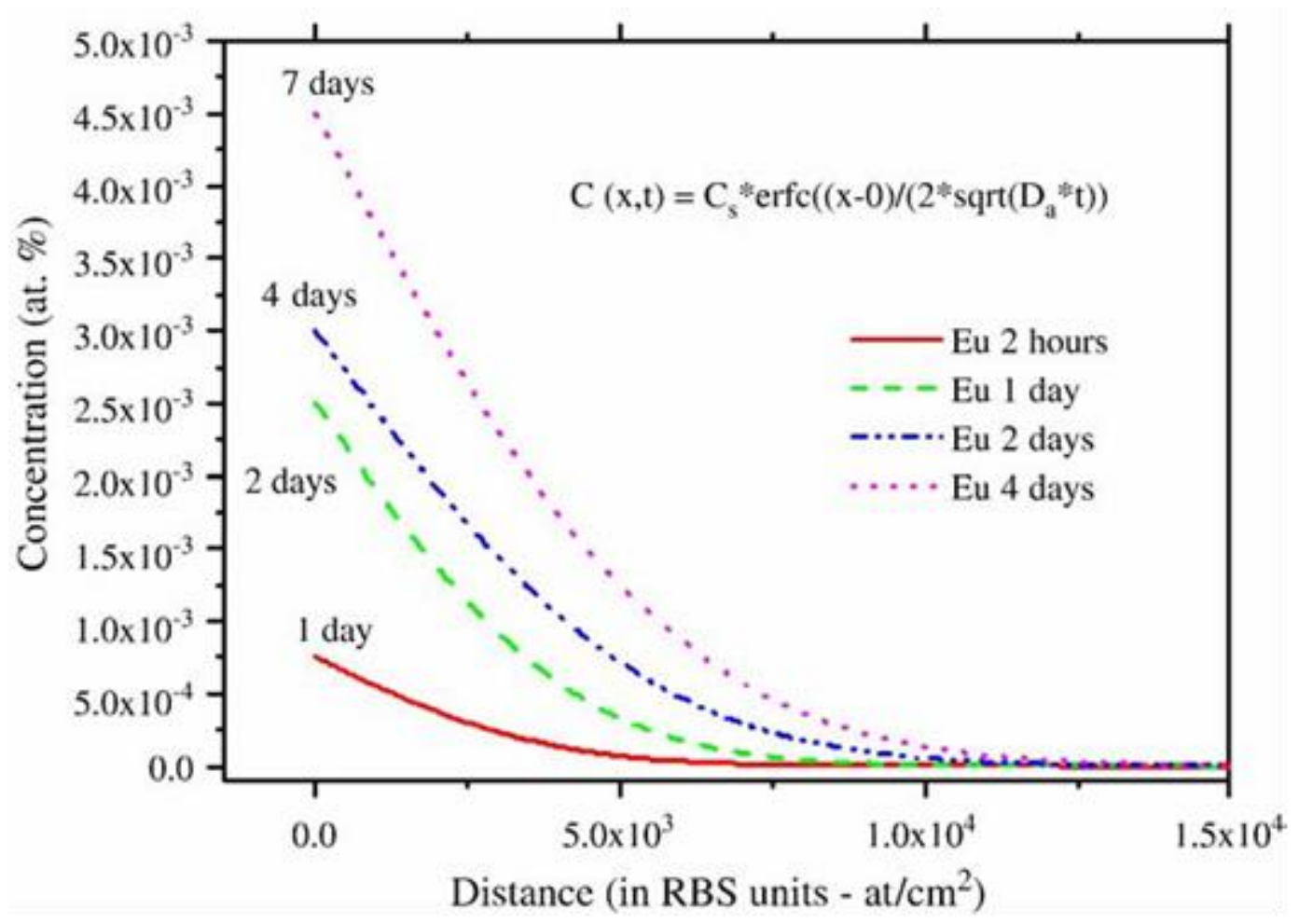

Figure 3. Diffusion constants extracted from RBS spectra. Reproduced from Figure 5 of Alonso et al, Applied Clay Science 43, 2009, 477-484 (ref [15]).

Fickian diffusion processes are also quantified by Ross et al [16] in an RBS study of defluorination mechanisms in poly(vinylidene) fluoride when treated with alkalis in the presence of phase transfer catalysts (see Figure 4). This work depends on the interpretation of the spectra in terms of molecular depth profiles (although this is not explicitly stated!). The chemical state of the elements is known because the defluorination process has been determined by other work (including XPS), and this chemical knowledge is used to relieve the ambiguity of these spectra. For a further thorough discussion of ambiguity see Jeynes et al [17].

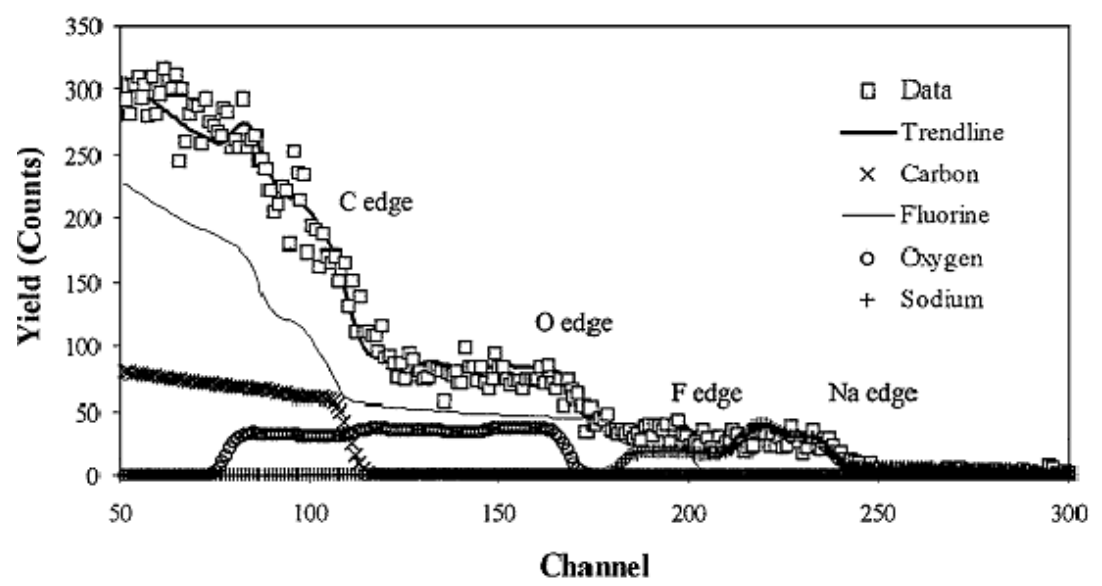

Figure 4. (Figure 4 of Ross et al 2000.) RBS of poly(vinylidene fluoride) exposed to alkali treatment in the presence of catalysts. Defluorination followed by oxidation reactions occur. 
Real-time processes can be followed by IBA since the analysis time can be very short. The earliest example of this we know is an in-situ study of the photodissolution of silver in an amorphous germanium selenide by Rennie et al (1986) [18] (see Figure 5). This work shows both the kinetics of the process and the way the mechanism switches when the thin continuous surface Ag film breaks up into islands before dissolving completely. The analysis is tricky because the RBS itself causes dissolution of the $\mathrm{Ag}$, and the total beam fluence must be restricted to $250 \mathrm{nC} / \mathrm{mm}^{2}$, with a fresh analysis spot used after every illumination. The spectra are then simply fitted with rectangular profiles, the low counting statistics in any case precluding any more detailed treatment. In Figure 5 the position of the surface signals of $\mathrm{Ag}$ and $\mathrm{Se}$ are shown, as is the position of the depth of the reaction interface at each time step. For short illumination times the Figure clearly shows the thickness of the surface Ag film reducing as the silver dissolves into the selenide. For long illumination times the reaction is complete. What is not shown are the intermediate times where the surface Ag signal keeps the same width but reduces its height, indicating an islanding reaction.

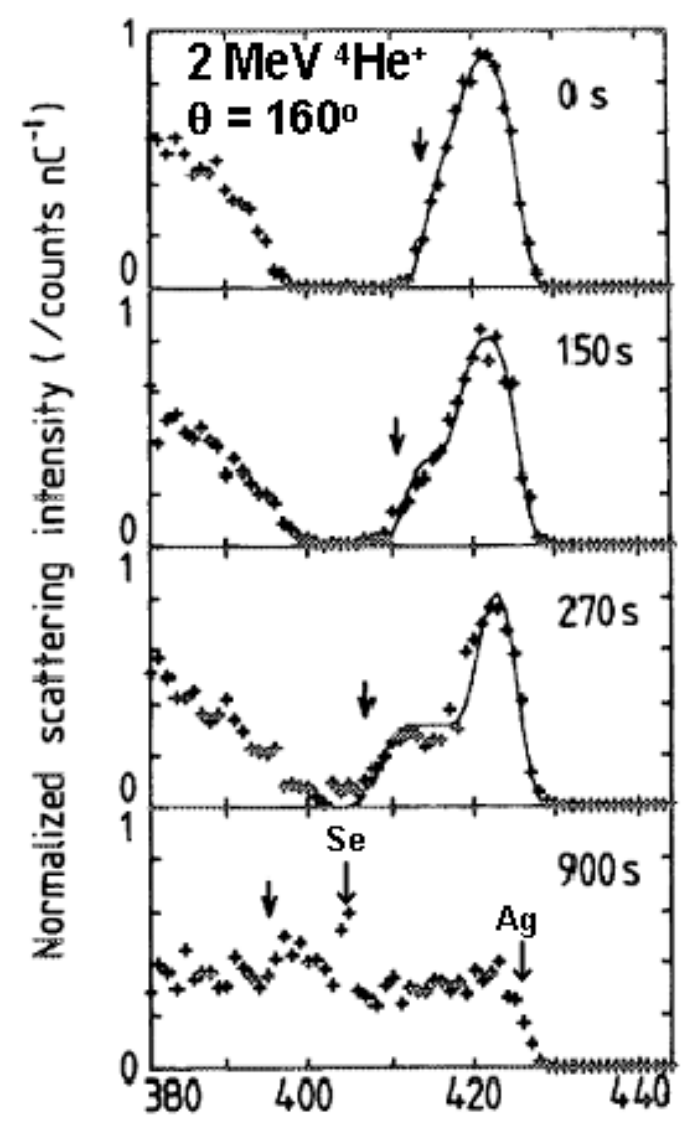

Channel no.

Figure 5. (Figure 1 of Rennie et al 1986.) RBS of a $38 \mathrm{~nm} \mathrm{Ag}$ film on a-GeSe $e_{2}$ exposed in-situ to $550 \mathrm{~nm}$ illumination at $4 \mathrm{~mW} / \mathrm{cm}^{2}$ for various times. 
Reaction kinetics are best followed in true real-time, as has been done in a series of recent reports. We will discuss the latest of these: Demeulemeester et al (2010) [19] (see Figures 6-8), who were investigating the kinetics of the formation of nickel silicides. What is of interest in such studies is the detection of the onset of the formation of phases, in this case the mono- and di-silicides. Conventionally, a series of anneals on different samples would be made, and then off-line analysis (RBS and other techniques) carried out to determine what had happened. The difficulty is that many samples are needed to determine annealing kinetics, and many more to precisely determine the position of the phase boundaries. This group has solved this first problem by collecting RBS spectra in real-time (every 30 seconds!) during the ramp-annealing. The question then is what to do with the voluminous data collected? Figure 6 shows a composite of all this data, which already looks suggestive. But of course, a quantitative analysis is desired. So they trained an artificial neural network (ANN) to recognise each spectrum! We should point out that this is considerably easier said than done, since a training set of 18000 spectra was required. This training set covered all possible variations of the spectra in this materials system, including reasonable roughness of the layers (we will discuss roughness in some more detail later). The quality of the solutions obtained by the ANN can be seen in Figure 7, and the solution of the whole dataset is shown in Figure 8 in terms of the proportion of each phase present as a function (effectively) of anneal temperature.

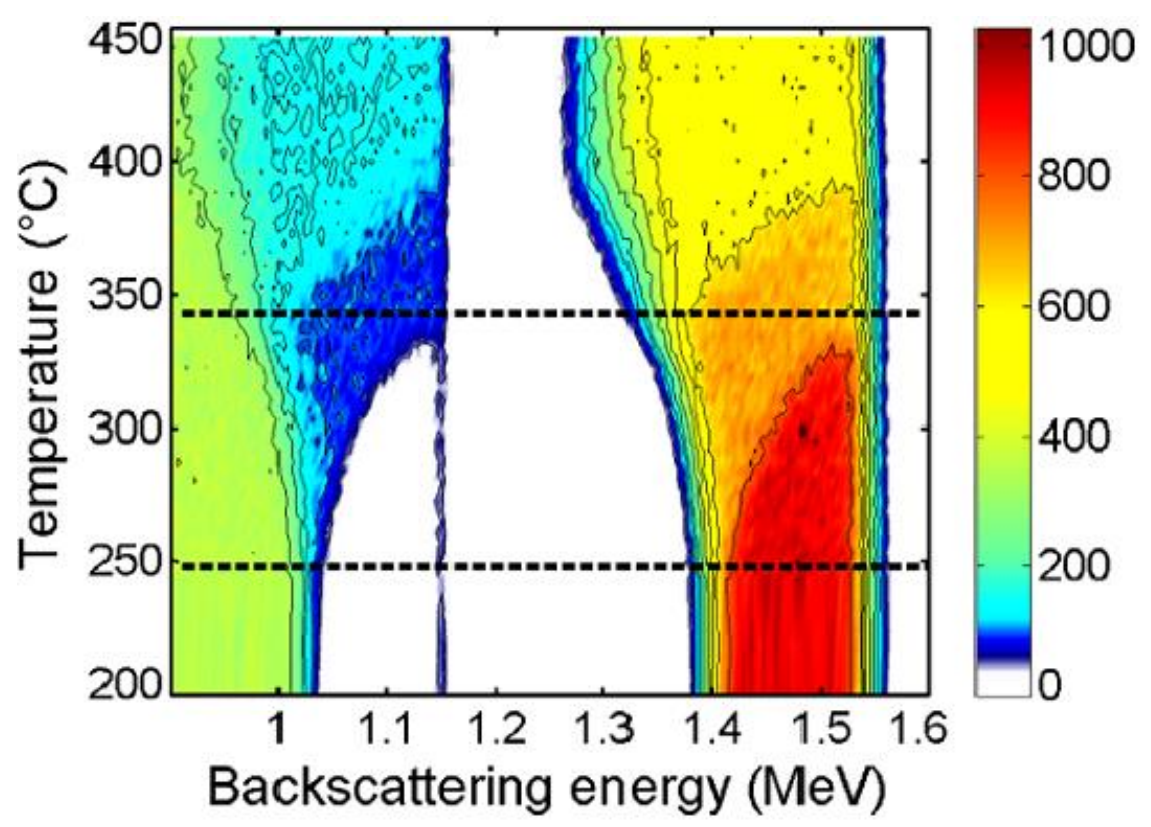

Figure 6. (Figure 2 of Demeulemeester et al 2010.) Composite of 250 RBS spectra taken during a Ni silicidation reaction of an $80 \mathrm{~nm}$ film on silicon, capped with $7 \mathrm{~nm}$ of Si and annealed at $2{ }^{\circ} \mathrm{C} / \mathrm{min}$. The colour code on the right shows spectral intensity in arbitrary units. The dashed lines indicate the initiation of the mono- and di-silicides. 


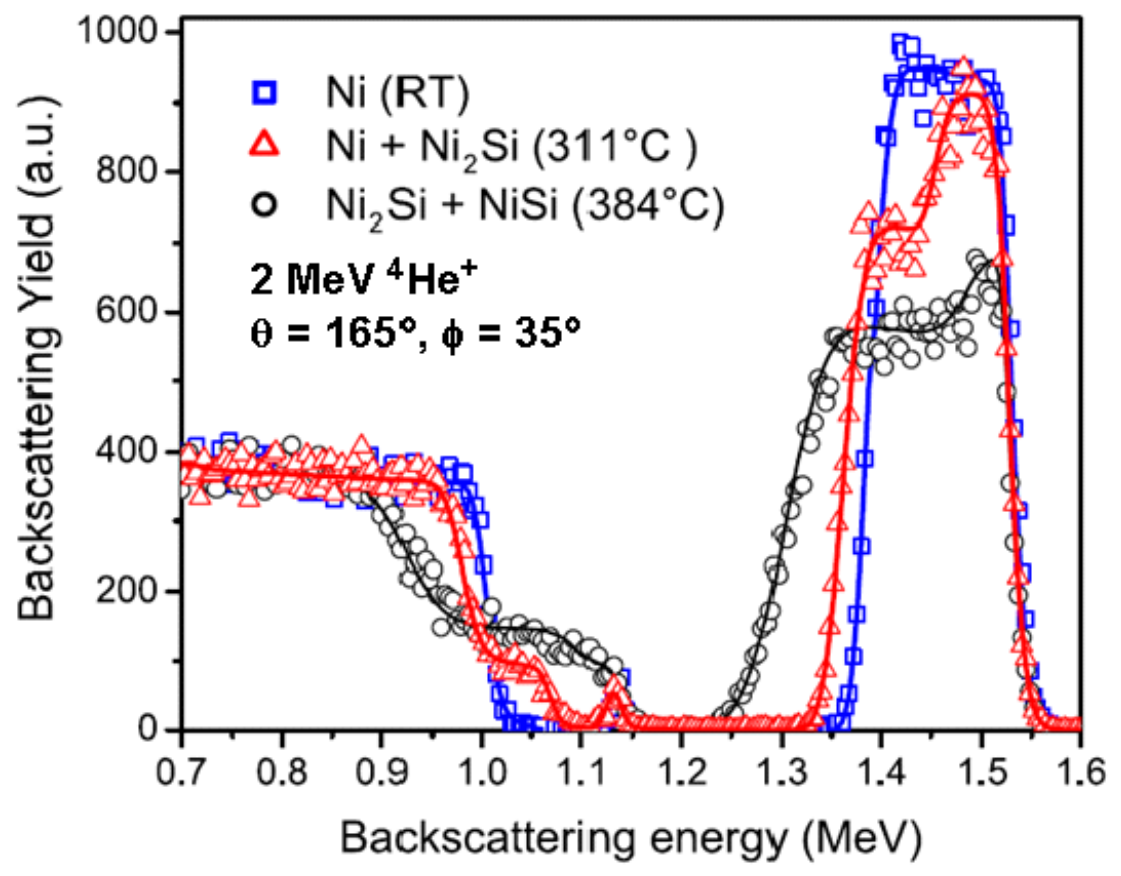

Figure 7. (Figure 4 of Demeulemeester et al 2010.) Three spectra from Figure 6 with solution by ANN.

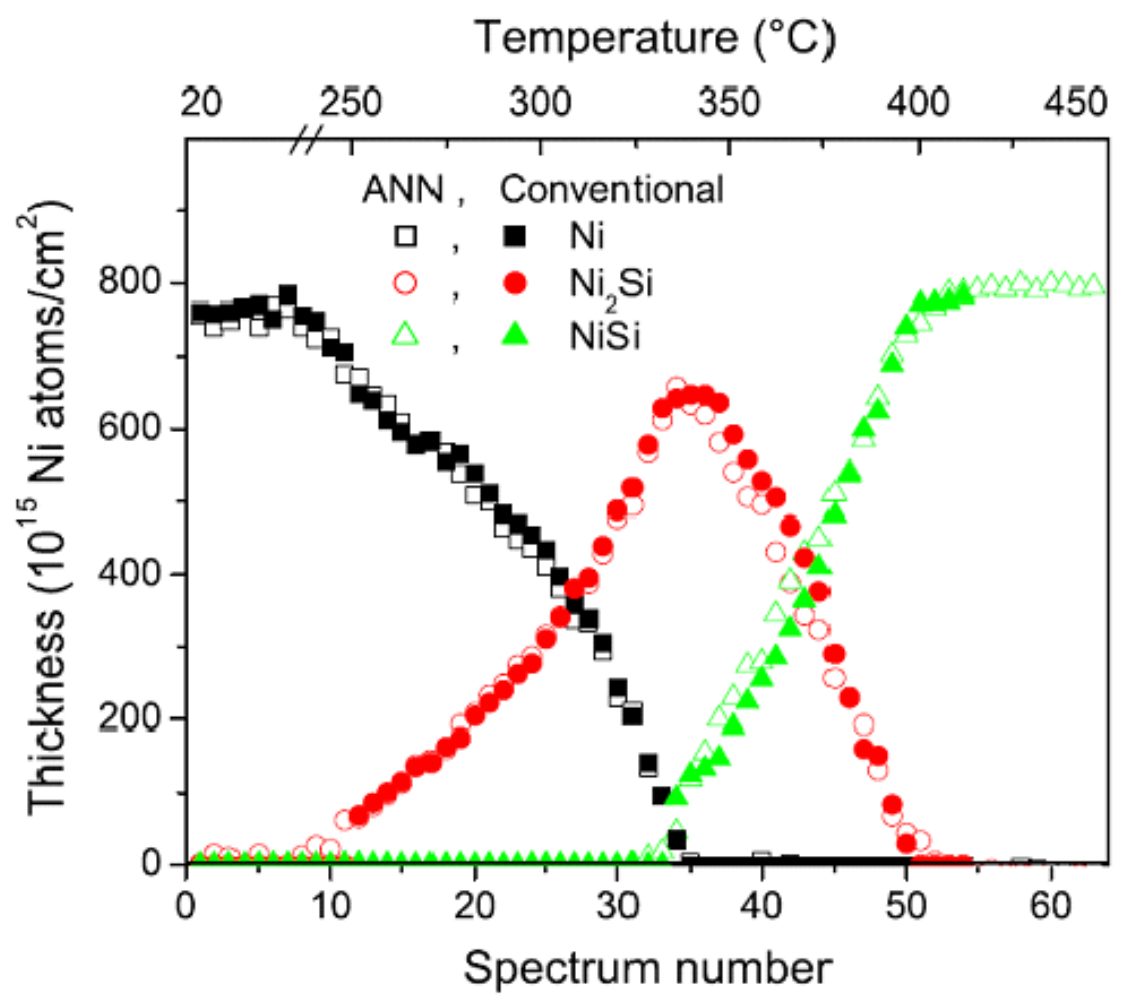

Figure 8. (Figure 3 of Demeulemeester et al 2010.) Solutions of spectra from Figure 6 over the whole annealing range, showing proportions of different phases, obtained both conventionally and by ANNs. 
We pause here for a digression on ANNs. If an artificial neural network can be trained to analyse RBS (and indeed, IBA data) with such precision, why do we need analysts at all? Surely we can now do "IBA without humans" [20]? ANNs give the (correct) answer completely automatically, and in effectively zero time. But an ANN is the ultimate black box. We do not (and probably cannot) know what exactly an ANN is doing. Therefore there are the dual problems of validation and of traceability (not to mention the problem of training the ANN in the first place!). We have to be able to check the correctness of the answer, and we have ultimately to be able to say what confidence we have in it. Fortunately, these are both very easy to supply: all we need to do is to give the ANN's solution back to a regular IBA code for refinement, and then we are back on solid ground since we know exactly how the regular codes work! This is what these workers did. Figure 8 shows the raw ANN result (for the whole dataset) directly compared with the result refined by the regular IBA code; the two are very similar! Thus, the ANN results can be treated as a "first-and-rather-good guess" to help the regular code with the solution. As a matter of fact, in this example the ANN could not precisely locate the phase boundaries, but what it could easily do is to determine the reaction kinetics (the activation energies and pre-exponential factors). And of course, these data are available in real-time, while the annealing is in progress.

Artificial neural networks are actually rather difficult to set up, and even when they are trained they are tricky to use. One always has to check that they are not giving nonsense. In principle one can train an (independent) ANN to recognise whether the input to an ANN is valid, that is, whether it is covered by the training set [21]. It is important to understand that an ANN can only interpolate within its training set - it cannot extrapolate. But this is a significant example because it shows that handling industrial quantities of data is entirely feasible, and moreover that it can be done at state-ofthe-art accuracy if necessary. If we wanted to, we could extend this sort of treatment to wider and wider classes, dramatically reducing the tedium of the routine analyses required from every IBA lab.

\section{Example of an optical multilayer}

The next example highlights something previously touched on: the usefulness of chemical information we may have about a sample; we also elaborate on our knowledge of the reliability of our results. Roughness is also important in the following examples.

Figure 9 shows a complex spectrum from a complex sample (see Jeynes et al (2000) [22]). It is an antireflection coating on glass, that is, alternating zirconia and silica $\left(\mathrm{ZrO}_{2}\right.$ and $\left.\mathrm{SiO}_{2}\right)$ layers on float glass: these oxides have strongly differing refractive indices giving the optical effect. The glass has many elements, and the zirconia naturally has a small amount of hafnium. This analysis cannot be made unless the problem is simplified to three logical elements, as opposed to the dozen or so real 
elements present. Again we have the problem of ambiguity (discussed at length in Jeynes et al, 2003 [17], and touched on in our discussion of Figure 4), that is, the information we would like access to is not unequivocally present in the data. One spectrum cannot usually determine the sample structure. Figure 9 is actually one spectrum from a pair: it was taken at normal incidence and there was another taken at $45^{\circ}$ incidence (not shown here). The two spectra look entirely different, and the two together determine the sample very much better than just one spectrum. But analysts must beware a gaping pitfall at their feet: the fact that the fit to the data is perfect is not a reason to think that the sample is perfectly known. The spectrum is ambiguous! A perfect fit means that the proposed profile is valid, not that it is true.

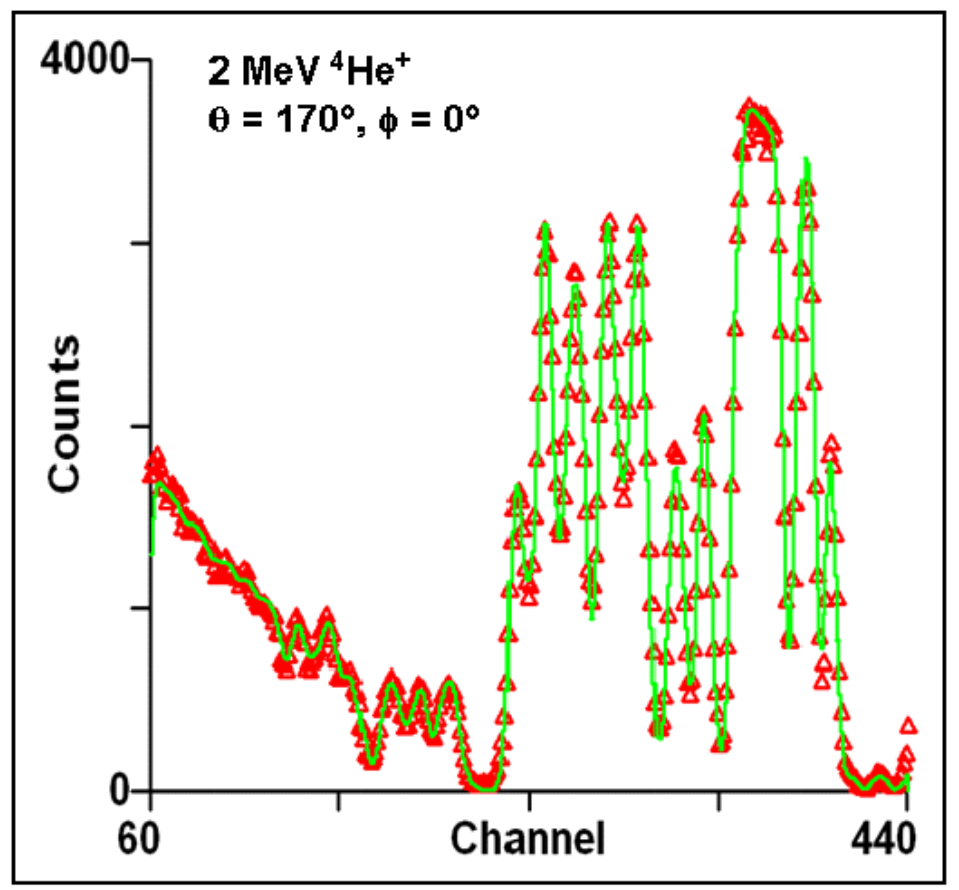

Figure 9. (From Figure 2 of Jeynes et al 2000.) Antireflection coating with alternate zirconia and silica layers on float glass. Normal incidence beam: the fit is shown as a line.

Even with two spectra the structure of the sample is completely ambiguous - until, that is, chemical information is introduced. Butler pointed out over twenty years ago [23] that our knowledge of the chemistry of the sample can always supplement our objective interpretation of IBA spectra obtained from the sample. In this case of course we are certain that the $\mathrm{Zr}$ and $\mathrm{Si}$ are fully oxidised - the optical properties tell us that! And we are also certain of the glass composition - centuries of glass technology assure us of that. Therefore we confidently use molecules as the logical elements of the analysis, and note that in our discussion of Figures 6,7 and 8 we already silently introduced molecules (the two phases of the silicide) to effect the analysis. 
The data in Figure 9 are actually analysed by the authors in an interesting way. They use simulated annealing, a mathematical global minimisation algorithm described elegantly in a Science article [24]. Simulated annealing was introduced into physics in 1953 [25] and into IBA analysis in 1997 ([26], and see the careful discussion in Jeynes et al, 2003 [17]). Simulated annealing is powerful in IBA since the user simply gives the data to the computer, without any initial guess of the depth profile which is extracted completely automatically. The method uses "Markov chain Monte Carlo" (MCMC) mathematics: this is described in the cited literature and can be summarised for this application as a systematic way of searching the space of possible solutions. And it is because the search is systematic that we can do statistics on it, using a Bayesian inference method [27]. This can be seen in Table 2, which shows extraordinarily precise layer thicknesses extracted from the RBS data down to depths of over a micron (and see Problem 2 for further meditations on the table).

\begin{tabular}{|c|c|c|c|c|c|c|c|c|}
\hline \multirow{2}{*}{$\begin{array}{l}\text { Layer } \\
\text { no. }\end{array}$} & \multirow{2}{*}{$\begin{array}{l}\text { Material } \\
\text { Air }\end{array}$} & \multirow{2}{*}{$\begin{array}{c}\mathrm{ZrO}{ }_{2} \text { content } \\
\%\end{array}$} & \multirow{2}{*}{$\begin{array}{l}\text { Last figure } \\
\text { error }\end{array}$} & \multicolumn{2}{|c|}{$\begin{array}{l}\text { Moasurod oquivalont thicknoss } \\
\text { for simulated annealing }\end{array}$} & \multicolumn{3}{|c|}{$\begin{array}{l}\text { Moasured oquival ont thicknoss } \\
\text { for MCMC }\end{array}$} \\
\hline & & & & $10^{15}$ atoms cm-2 & $\mathrm{nm}$ & $10^{15}$ atoms cm-2 & $\mathrm{nm}$ & Error $(\mathrm{nm})$ \\
\hline 0 & $\mathrm{Au}$ & 35.3 & 5 & 24.3 & 4.1 & 35.2 & 5.96 & 0.02 \\
\hline 1 & $\mathrm{ZrO}_{2}$ & 91.5 & 13 & 154.0 & 18.8 & 141.2 & 17.2 & 0.6 \\
\hline 2 & $\mathrm{SiO}_{2}$ & 2.7 & 2 & 436.2 & 72.7 & 411.9 & 68.6 & 0.8 \\
\hline 5 & $\mathrm{ZrO}_{2}$ & 95.0 & 6 & 1045.2 & 127.3 & 1044.4 & 127.2 & 1.7 \\
\hline 6 & $\mathrm{SiO}_{2}$ & 1.3 & 4 & 706.6 & 117.8 & 689.8 & 115.0 & 2.4 \\
\hline 7 & $\mathrm{ZrO}_{2}$ & 73.9 & 43 & 169.5 & 20.6 & 182.8 & 22.3 & 1.8 \\
\hline 8 & $\mathrm{SiO}_{2}$ & 3.5 & 7 & 533.6 & 88.9 & 533.6 & 88.9 & 2.5 \\
\hline 9 & $\mathrm{ZrO}_{2}$ & 53.1 & 25 & 169.2 & 20.6 & 168.1 & 20.5 & 1.2 \\
\hline 14 & $\mathrm{SiO}_{2}$ & 7.5 & 13 & 428.7 & 71.4 & 442.6 & 73.8 & 2.0 \\
\hline 15 & $\mathrm{ZrO}_{2}$ & 76.0 & 18 & 269.9 & 32.9 & 299.2 & 36.4 & 1.8 \\
\hline 16 & $\mathrm{SiO}_{2}$ & 13.0 & 12 & 408.6 & 68.1 & 389.2 & 64.9 & 2.5 \\
\hline 17 & $\mathrm{ZrO}_{2}$ & 74.7 & $1 \overline{3}$ & 275.7 & 33.6 & 246.5 & 30.0 & 1.3 \\
\hline 18 & $\mathrm{SiO}_{2}$ & 17.0 & 8 & 525.6 & 87.6 & 531.8 & 88.6 & 1.8 \\
\hline \multirow[t]{2}{*}{19} & $\mathrm{ZrO}_{2}$ & 26.0 & 6 & 106.0 & 12.9 & 118.5 & 14.4 & 0.5 \\
\hline & & & & tâl: & 1137 & & 1136 & \\
\hline
\end{tabular}

Density assumed: $\mathrm{ZrO}_{2}=5.6 \mathrm{~g} \mathrm{cc}^{-1}\left(821 \times 10^{20}\right.$ atoms cc$\left.{ }^{-1}\right)$. The simulated annealing calculation was the starting point for the MCMC calculation.

Table 2. (Table 2 of Jeynes et al 2000.) Solution of antireflection coating (see Figure 9) with uncertainties from a Bayesian inference analysis (see text).

\section{Example of intermixing analysis}

Figure 10 shows an interesting example of $\mathrm{Fe} / \mathrm{Si}$ multilayer samples with and without ion-beaminduced intermixing, and Problem 3 explores this further. The idea is to try to find some feasible way to make amorphous iron disilicide, which is a semiconductor that could become an important thin film 
solar photovoltaic material if technological problems can be overcome [28]. Much ion beam analysis is aimed at elucidating the behaviour of materials in a variety of processes to enable some approaches to be ruled out and others to be developed effectively. This example is included as "one that didn't work", since materials scientists usually need to understand these cases rather better than the successful ones!
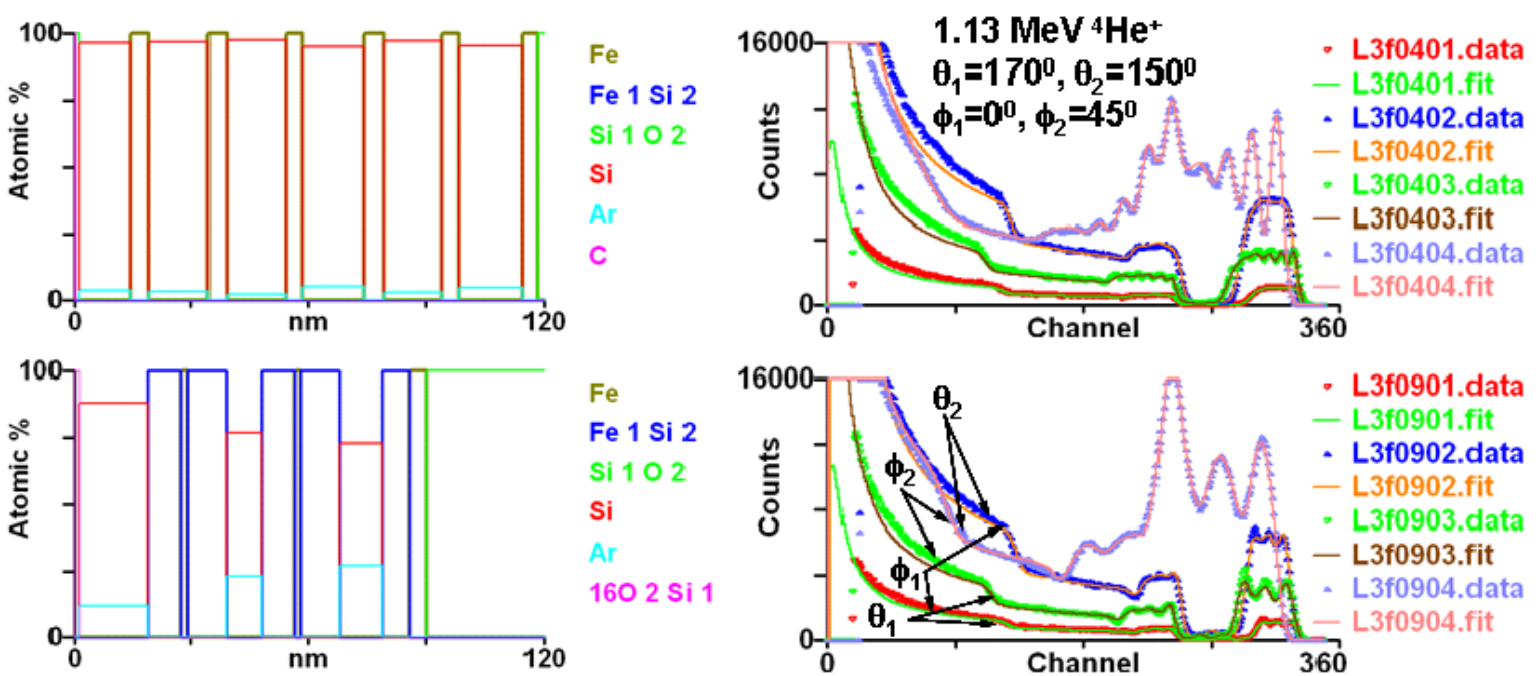

Figure 10 (unpublished). Above: 12-layer Fe-Si multilayer on quartz as deposited.

Below: 6-layer Fe-Si multilayer, implanted with $200 \mathrm{keV} 5.15^{15} \mathrm{Fe} / \mathrm{cm}^{2}$.

Left: depth profiles derived from RBS data. Right: collected data for two detectors and two beam incidence angles. For tilted incidence the exit angle for detector 1 (Cornell geometry) is about $45^{\circ}$ but for detector 2 (IBM geometry) it is $75^{\circ}$.

We intended to lay down pure Fe and Si layers (by magnetron sputtering) of the correct thicknesses, and then intermix these by ion bombardment at elevated temperatures. The nuclear displacements during ion implantation ("ballistic mixing") will stir up (mix) the sample to an extent that can be calculated by the standard Monte Carlo codes (SRIM: see www.srim.org). But extra radiationenhanced diffusion is also expected, that is, diffusion in excess of the equilibrium diffusion expected at the implantation temperature. Ion implantation is a strongly non-equilibrium process, and radiation-enhanced diffusion is a consequence of this. And indeed, strong intermixing is observed. However, the analysis showed that the presence of $\mathrm{Ar}$ (from the sputter-deposition) poisons the development of the silicide.

The coating includes layers of only a few nm thick, which is challenging for standard RBS. Fe and $\mathrm{Si}$ are sequentially sputter-deposited, but although the close-packed metal does not incorporate significant Ar, the more open Si layers incorporate quite a lot. During the development of the silicide the $\mathrm{Ar}$ is swept out to the remaining unreacted $\mathrm{Si}$, preventing the completion of the process. 
In this analysis the RBS of course objectively sees only the Fe and Si (and Ar) elemental profiles, but it is valuable to be able to interpret these elemental profiles as profiles of the phases present, as we have already done for the data in Figures 4, 6-8 and 9. Note that the only minor element present, with the exception of a little surface oxide, is Ar, which is quite well determined in this analysis. But the high quality of the fits to the data seen in Figure 10 is only obtained when the straggling is correctly calculated, firstly by the detailed physics incorporated in Szilágyi's DEPTH code [29] (this code or its equivalent is incorporated in both the "new generation" codes, SIMNRA and DataFurnace); and secondly by taking account of the extra straggling generated by "roughness", in this case layer thickness inhomogeneity (which the "new generation" codes also incorporate). Note that all four spectra (two separate detectors, two different beam incidence angles) are equally well fitted, showing that these profiles are well-determined.

\section{Example of corrosion analysis}

Pascual-Izarra et al (2007) have given a first and powerful demonstration of the simultaneous and self-consistent use of particle scattering and photon emission [30]. They analysed for conservation purposes an important cultural heritage artefact from the Louvre Museum, one of the earliest photographs in the world (see Figure 11). It was corroding, and precise details were required. The external microbeam of the AGLAE accelerator sited at the Louvre was used to non-destructively depth profile selected areas of the photograph, and the results are shown in Figure 12. This figure shows RBS, EBS and PIXE data, and we emphasise that the sample cannot be elucidated by any one of these spectra handled separately; the depth profile also shown in the figure is obtained only when all the data are analysed self-consistently.

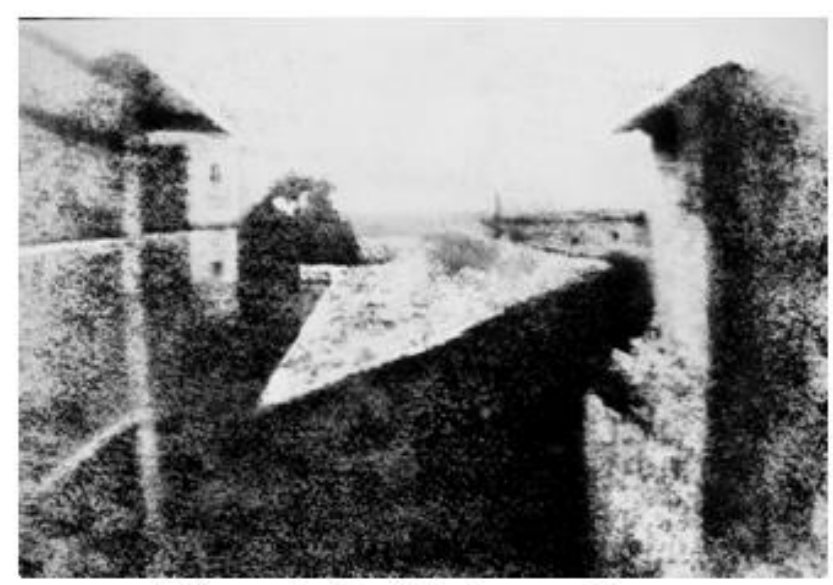

\section{Niépce's first Heliography: \\ Paysage à Saint-Loup de Varennes (1827)}

Figure 11. Specimen from the collection of the Louvre Museum (Paris). 

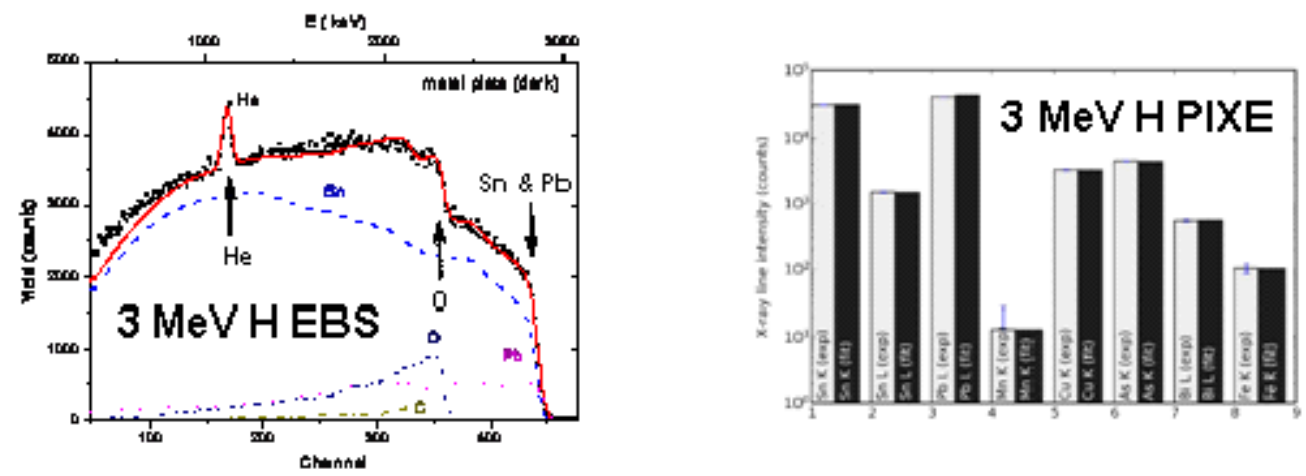

Fitted RES spectrum for $3 \mathrm{MeV} \mathrm{H}^{+}$b erm on the dack sot (corroded ax a). Calcubted partial spectra for anch elem entar e also showr

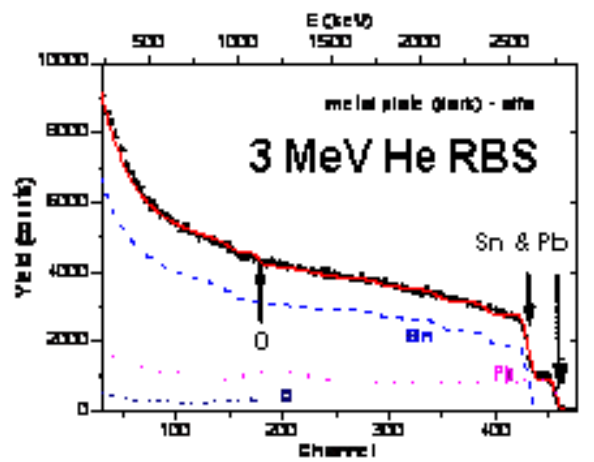

Fitted RES spectum for $3 \mathrm{MeV}$ the $\mathrm{b}$ am on the dark spot (corroded area). Calculated partinl spextar for each elem enta re abo shown

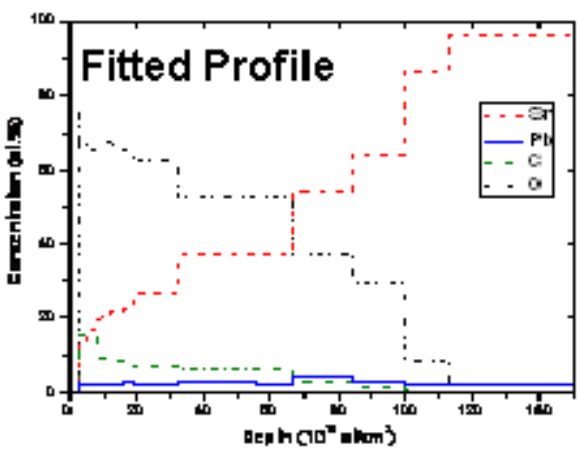

Corcertation protiles for the dark sp ot icorrod ed aI a) , $\pi$ s obtain ed from $a$ simultreous fit to $3 \mathrm{lWeV}$ protor PLE and RBS, ara $3 \mathrm{MeV}$ alp ha RES.

Figure 12. (Pascual-Izzara et al 2007.) RBS, EBS and PIXE data from a corroded region of Niépce's heliograph (see Figure 11) together with the fitted profile extracted from the data using DataFurnace (NDF). PIXE data is pre-processed by GUPIX to obtain characteristic line areas.

First (and not shown), an uncorroded region was analysed to obtain the bulk composition (homogenous in depth). The authors demonstrated that the PIXE and RBS/EBS results agreed. In this case of course, either the particle spectra or the PIXE spectrum can separately yield the bulk composition. In this case the PIXE detector must be properly calibrated, the RBS gives the Pb/Sn composition at the surface, and the EBS demonstrates for the analysis depth (over 15 microns) that the composition is uniform. Differential PIXE (whose information depth exceeds 30 microns) can be used to show the composition is uniform to greater depth. XRF can go even deeper. Note that in this case, although the $\mathrm{Pb} \mathrm{K}$ lines could not be excited, both the Sn K and L lines were used, which itself is a form of differential PIXE.

For the corroded sample however, the modified surface layer thickness is not great enough to easily use differential PIXE (and in any case, GUPIX does not currently support this sort of depth profiling). But in this case the EBS immediately shows that the sample is oxidised, with an oxidation front 
penetrating the sample irregularly to a depth of around $10^{20}$ atoms $/ \mathrm{cm}^{2}$ (what linear thickness this represents depends on the oxidation states present). The trouble is that the EBS cannot tell which component is oxidising, the RBS can only distinguish $\mathrm{Pb}$ and $\mathrm{Sn}$ at the surface, and the PIXE has no depth resolution. The spectra, separately, are entirely ambiguous, but when all the data are put together the profile is unambiguous. The clean sample determines the substrate (underlying) composition (a boundary condition), the RBS determines the $\mathrm{Pb}: \mathrm{Sn}$ ratio at the surface (the other boundary condition), the EBS determines the O profile, and the PIXE determines the relative $\mathrm{Pb}: \mathrm{Sn}$ in the profile calculated self-consistently. Of course, the PIXE also determines the total quantity of the minor and trace elements, which can also be profiled in the same way. In this way some oxidation models can be demonstrated to be consistent with the data, and other models inconsistent.

We point out here some interesting features of these data. An external beam was used, that is, the proton beam was brought out to air to analyse these valuable object, which of course cannot be cut to fit inside a vacuum chamber, and in any case are usually considered too delicate to be subjected to a vacuum system. MeV charged particle beams are very penetrating and have useful working distances of centimetres. To increase working distances, and reduce effects both of X-ray absorption (giving more sensitivity for the lower X-ray energy lines) and of backscattered particle energy loss, a He flush around the beam paths is used. This He can be seen in the EBS spectrum! Note that the He signal is fitted very well, showing that the particle detector is aligned and collimated correctly. If $\mathrm{He}$ is not used, then $\mathrm{Ar}$ (present at about $1 \%$ in air) will be seen in the PIXE spectrum, and $\mathrm{O}+\mathrm{N}$ in the BS spectra.

Note that, at these energy proton beams, all elements to at least Fe have non-Rutherford scattering cross-sections, and note also that evaluated cross-sections (that is, known very well for any geometry) are now available for most of the important elements [31]. Clearly, to use EBS the scattering crosssections must be known, but at present the RBS-EBS boundary is not well known, even in principle. The MRS Handbooks [32] have chapters on the Coulomb barrier, potentially extremely misleading since "the Coulomb barrier" is not a well defined idea [33]. But at this energy the BS signals for the heavy elements $\mathrm{Sn}$ and $\mathrm{Pb}$ are Rutherford. Note that with a $3038 \mathrm{keV}{ }^{4} \mathrm{He}$ beam there is a strong EBS resonance for surface $\mathrm{O}$, so that if a slightly increased beam energy had been used the surface $\mathrm{O}$ would have been visible even for the "RBS" spectrum.

In Figure 12, clearly the $3 \mathrm{MeV}$ RBS/EBS and the $3 \mathrm{MeV}$ PIXE spectra were collected simultaneously using the proton beam. For $3 \mathrm{MeV}$ He the PIXE spectrum (available as well as the BS spectrum) is not shown for clarity, but it is worth pointing out that this is another way to do differential PIXE since the information depth for $3 \mathrm{MeV}$ He-PIXE is much less than for $3 \mathrm{MeV} \mathrm{H}$ PIXE. 


\section{Example of 3D analysis for geology}

Our last example is of the so-called Darwin glasses (see Bailey et al, 2009) [34] which are impact glasses resulting from a meteor strike 800000 years ago near Mt Darwin in Tasmania. The geologist studying these, subsequently used one of his Darwin glass samples as an amorphous standard for setting up his XRD kit, and was astonished to see the diffraction spots of quartz. These crystals unexpected in a glass! - turned out to be inside inclusions in the glass. But the nature of these inclusions was entirely unknown.

$\mu$ PIXE (Figure 13) shows that the samples are very heterogeneous, and EBS analysis unequivocally demonstrated them to be carbonaceous from the ${ }^{12} \mathrm{C}(\mathrm{p}, \mathrm{p}){ }^{12} \mathrm{C}$ resonance at $1734 \mathrm{keV}$ (Figure 14). This result initially baffled the geologists, for whom such a sample was unprecedented. Figure 15 shows that the IBA data could be completely and quantitatively analysed without any presupposed model despite the heterogeneity.
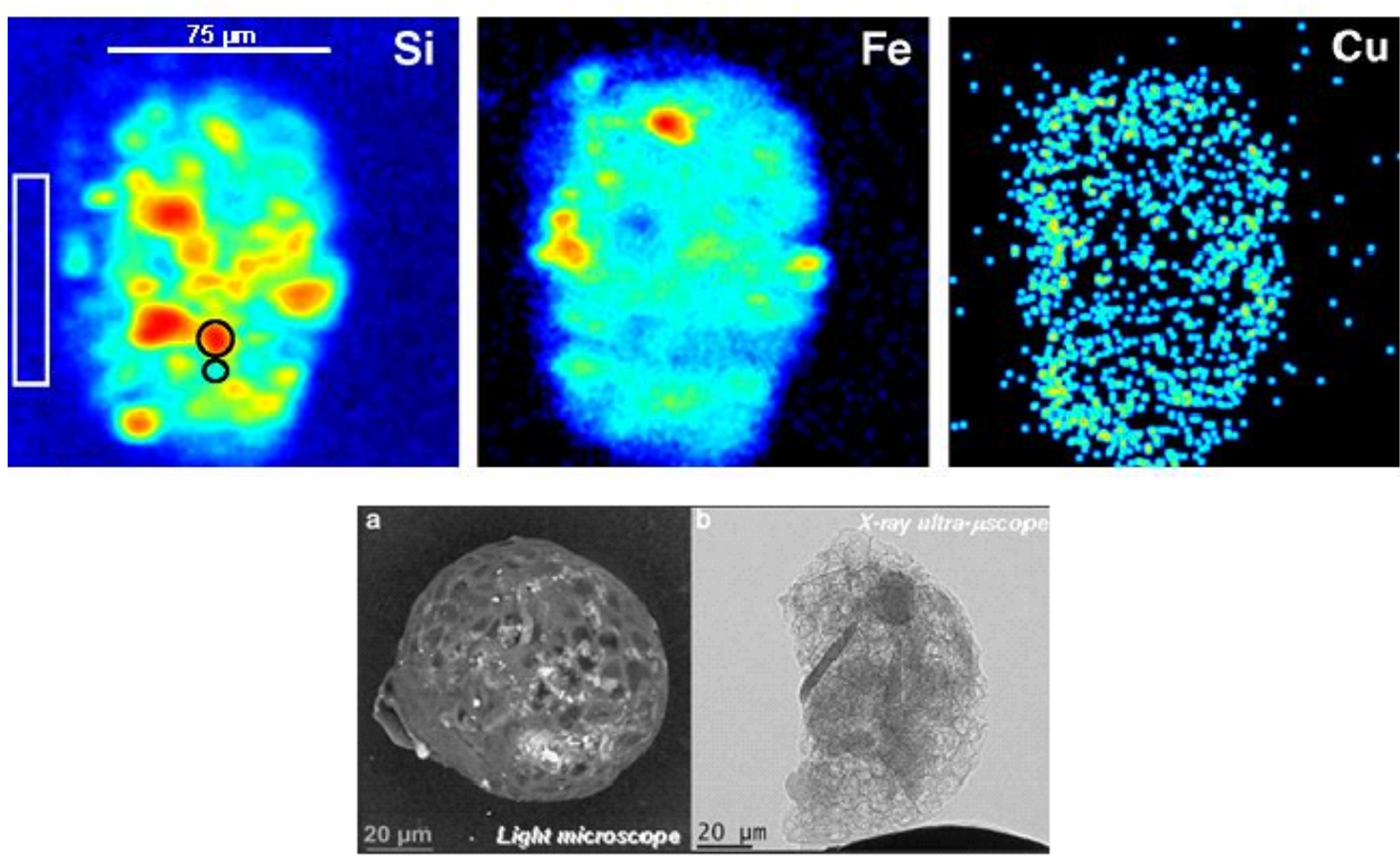

Figure 13. Above (Figure 1 of Bailey et al 2009): PIXE maps for $\mathrm{Si}, \mathrm{Fe}$ and $\mathrm{Cu}$ of Darwin glass set in resin, showing radical inhomogeneity. Below (by courtesy of $\mathrm{T} \mathrm{K}$ Howard): Light microscope and X-ray radiographs of typical Darwin glass inclusions. 

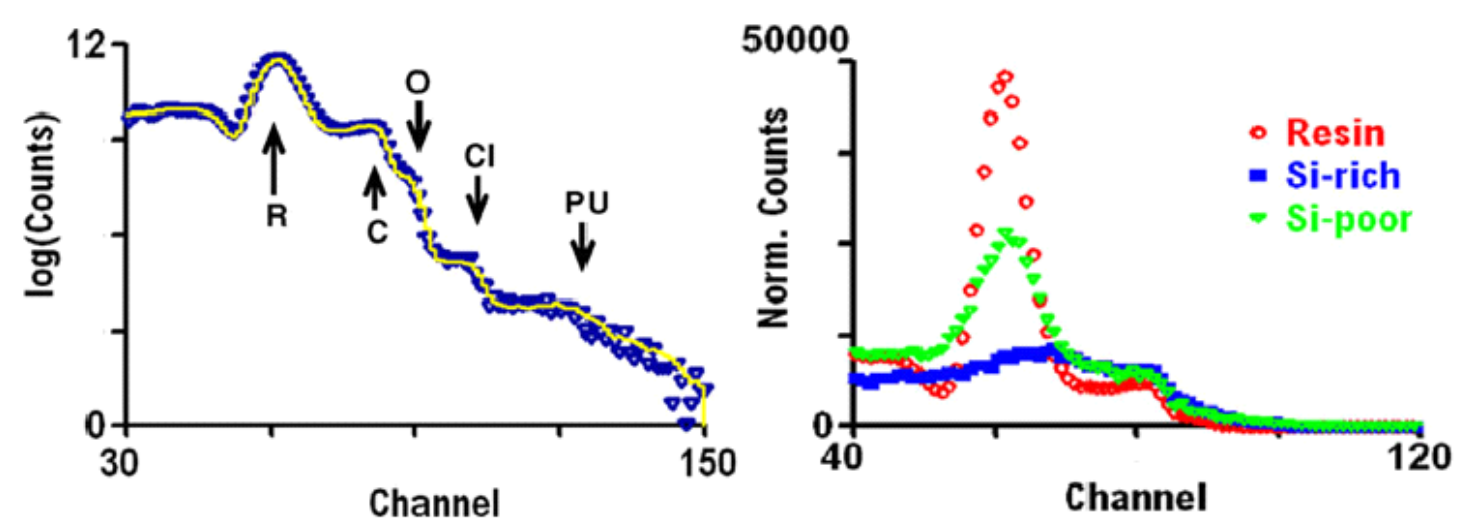

Figure 14. Left (Figure 4 of Bailey et al 2009): EBS for resin - see rectangle in Figure 13 (Si PIXE). The surface signals for $\mathrm{C}, \mathrm{O}, \mathrm{Cl}$ are marked, as are the pileup signal and the signal due to the ${ }^{12} \mathrm{C}(\mathrm{p}, \mathrm{p}){ }^{12} \mathrm{C}$ resonance at $1734 \mathrm{keV}$ (note $\log _{\mathrm{e}}$ scale). Right (Figure 3 of Bailey et al 2009): Charge-normalised EBS for all marked areas in Figure 13.
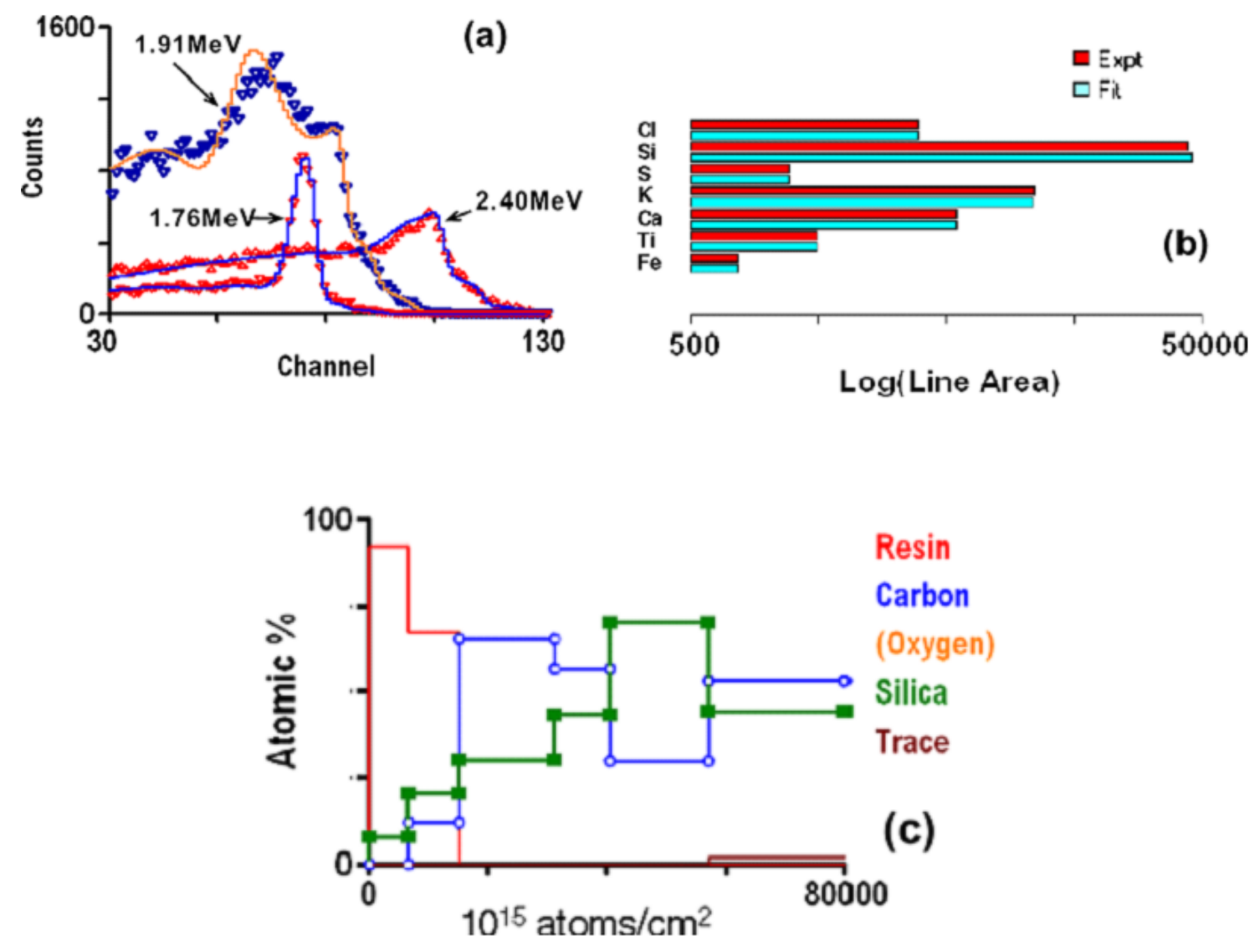

Figure 15. Analysis of a Si-rich area of a Darwin glass (see region marked in Figure 13 and spectrum in Figure 14 (Figure 6 of Bailey et al 2009): (a) EBS spectra at various energies; (b) 2.4 MeV PIXE; (c) fitted depth profile consistent with the data.

Microbeam PIXE/BS demonstrated the great heterogeneity of the samples both laterally and in depth; this sort of mapping microbeam data effectively gives two 3D data cubes, with (say) $128 \times 128$ pixels 
and a PIXE and BS spectrum pair for each pixel. The data cube pair, intractable as it stands, can be analysed into its principal components by using some multivariate image analysis program, then each pair of BS/PIXE spectra can be analysed to give an explicit depth profile, from which the 3D representation of the sample could be reconstructed.

What is also interesting about these data is that the heterogeneities are radical, that is, there are precipitates of one material (quartz) in another (the carbonaceous matrix). On the face of it, one does not expect to be able, by IBA, to distinguish a material with precipitates from a material with a uniform (average) composition. But Stoquert \& Szörenyi (2002) [35] demonstrated that in fact the density variation in a material will measurably affect the straggling of the probe ion beam as it penetrates the sample. And we can use sharp non-Rutherford resonances in the elastic scattering cross-section as markers for the straggle as a function of depth. This was used by Tosaki (2006) [36] to distinguish different forms of carbon. It is the behaviour of the EBS resonance for ${ }^{12} \mathrm{C}(\mathrm{p}, \mathrm{p}){ }^{12} \mathrm{C}$ at $1734 \mathrm{keV}$ that allows us to prove the presence not only of carbon but also of $\mathrm{SiO}_{2}$ precipitates in these inclusions.

It is worth elaborating this example, since the details are rather arcane. Figure 14 shows, first, the EBS spectrum from the resin in which the geological sample is embedded in the standard mineralogical procedure. This spectrum is readily interpreted, and the most prominent feature in it is clearly the signal of the $1734 \mathrm{keV}$ resonance in the EBS cross-section for carbon. The spectrum is shown on a $\log$ scale, which makes visible the signal of $\mathrm{Cl}$, a minor element. Note also that the pileup signal is not negligible, even for a microbeam analysis which usually uses a very restricted beam current. The $\mathrm{C}$ resonance gives both a greatly enhanced and also (in the tail of the resonance) a greatly reduced EBS signal, following the cross-section function. But when the spectra from the inclusion itself are analysed, the resonance shape seems smeared out. In the Si-rich region we might expect that the reason for this is that there is just not that much $\mathrm{C}$ present, but this is clearly not true in the Si-poor region, which still has a very pronounced $\mathrm{C}$ resonance signal. But it is the wrong shape!

This behaviour is emphasised when the Si-rich region is analysed in more detail (Figure 15). Clearly, $\mathrm{C}$ is present as a major element judging from the $1.76 \mathrm{MeV}$ EBS spectrum, where the EBS crosssection for surface $\mathrm{C}$ is enhanced (although this is best interpreted as some resin present on the surface). But at some depth the $\mathrm{C}$ concentration is markedly reduced, as the $1.91 \mathrm{MeV}$ EBS spectrum shows (this is the same spectrum shown in Figure 14). But the spectral shape is also very different from what we expect. The correct interpretation of this spectrum is that extra straggling is introduced by the fact that the large amount of $\mathrm{Si}$ present is in the form of silica precipitates, giving a strongly inhomogeneous density. The algorithm implemented in the IBA code approximates this extra straggling quite well, even for this extreme case; and, when the information in the PIXE data on the Si 
content is included, an unequivocal depth profile is obtained. Note that a self-consistent analysis is made of the multi-energy, multi-technique data.

This last example points towards tomography. X-ray tomography (XR-T) is already established (see an example in Figure 14), and STIM-T is an almost equivalent (and solved) problem [37]. Great strides have also been made towards a PIXE-T [38], which is qualitatively much more complex than either STIM-T or XR-T. We have shown that in principle IBA-T (that is, using the BS signals as well as the PIXE signals) is already achievable, and should be significantly more efficient (and therefore much faster!) than pure PIXE-T since a single slice already has (nearly) complete 3D information. This is important since tomography is rather slow, and its importance is increased since it seems that beam damage limits the use of a pure PIXE-T for important classes of samples.

\section{Conclusions}

Backscattering analysis (Rutherford or not) is a naturally powerful technique for thin film depth profiling. There are modern IBA codes powerful enough to untangle the most complicated spectra, and these methods have been used effectively throughout thin film materials science. But the power of IBA increases dramatically when PIXE is added to the analyst's toolbox. Note that for all IBA, PIXE and BS data are always present together, so the data collection itself does not take longer. PIXE data gives the analyst an integral view of the sample, with high elemental specificity and sensitivity, almost perfectly complementing the depth profiling BS which mixes up mass and depth information. We have shown two examples of an integrated PIXE/BS analysis; the first of a rather simple case, but the second of a highly complex case involving not only elemental inhomogeneity but also partition into discrete phases inside the sample. We showed how even this case could be handled successfully by IBA.

Looking to the future, we have also shown that an IBA-tomography is feasible which is likely to be more than an order of magnitude faster than a pure PIXE-tomography. Whether or not this is ever realised, it is clear that microbeam IBA is capable of a 3D analysis that is today largely untapped and that shows immense promise.

We conclude that, where backscattering and PIXE individually are powerful and versatile techniques, "Total IBA" (that is, an integrated treatment of BS/PIXE data) has a synergy that effectively completely generalises depth profiling IBA. Twenty-first century thin film depth profiling needs Total IBA. 


\section{References}

[1] Glossary: $\boldsymbol{I B} \boldsymbol{A}=$ "ion beam analysis"; $\boldsymbol{R} \boldsymbol{B S}=$ "Rutherford backscattering"; $\boldsymbol{E} \boldsymbol{B S}=$ "elastic (non-

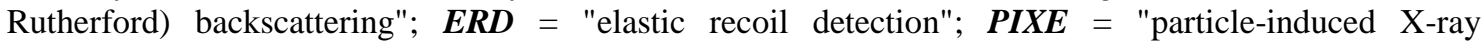
emission"; $\boldsymbol{N R P}=$ "nuclear reaction profiling"; $\boldsymbol{M E I S}=$ "medium energy ion scattering"; $\boldsymbol{L E I S}=$ "low energy ion scattering"; $\boldsymbol{A N N}=$ "artificial neural network". GUPIX = "Guelph PIXE program", that is, the widely used program by Campbell \& Maxwell. "IBM geometry" = detector in the horizontal plane. "Cornell geometry" = detector in the vertical plane. Neither IBM in Yorktown Heights nor Cornell University at Ithica, New York now have IBA laboratories.

[2] Jenneson PM, Clough AS, Hollands R, Mulheron MJ, Jeynes C, Profiling chlorine diffusion into ordinary Portland cement and pulverized fuel ash pastes using scanning MeV proton micro-PIXE, Journal of.Material.Science.Letters 17(14), 1998, 1173-1175.

[3] Riggs PD, Clough AS, Jenneson PM, Drew DW, Braden M, Patel MP, ${ }^{3} \mathrm{He}$ ion-beam analysis of water uptake and drug delivery, Journal of Controlled Release 61, 1999, 165.

[4] Smith RW, Massingham G, Clough AS, In 10th Symposium on Radiation Measurements and Applications Elsevier Science Bv: Ann Arbor, Michigan, 2002, 582.

[5] Clough AS, Collins SA, Gauntlett FE, Hodgson MR, Jeynes C, Rihawy MS, Todd AM, Thompson RL, In situ water permeation measurement using an external ${ }^{3} \mathrm{He}^{2+}$ ion beam, Journal of Membrane Science 285, 2006, 137-143.

[6] Gall TP, Lasky RC, Kramer EJ, Case II diffusion: effect of solvent molecule size, Polymer 31, 1990, 1491-1499.

[7] Cherniak DJ, Watson EB, Grove M, Harrison TM, Pb diffusion in monazite: a combined RBS/SIMS study, Geochim Cosmochim Acta 68, 2004, 829.

[8] Thomas NL, Windle AH, A theory of Case II diffusion, Polymer 23, 1982, 529-542.

[9] Shearmur TE, Clough AS, Drew DW, van der Grinten MGD, Jones RAL, Interdiffusion of deuterated and protonated poly(methyl methacrylate), Polymer 39, 1998, 2155-2159.

[10] Geoghegan M, Jones RAL, van der Grinten MGD, Clough AS, Interdiffusion in blends of deuterated polystyrene and poly(a-methylstyrene), Polymer 40, 1999, 2323-2329.

[11] Composto RJ, Mayer JW, Kramer EJ, White DM, Fast mutual diffusion in polymer blends, Physical Review Letters 57, 1986, 1312-1315.

[12] Thompson RL, McDonald MT, Lenthall JT, Hutchings LR, Solvent accelerated polymer diffusion in thin films, Macromolecules 38, 2005, 4339-4344.

[13] Composto RJ, Kramer EJ, Mutual diffusion studies of polystyrene and poly(xylenyl ether) using Rutherford backscattering spectrometry, Journal of Materials Science 26, 1991, 2815-2822.

[14] Moncoffre N, Barbier G, Leblond E, Martin Ph, Jaffrezic H, Diffusion studies using IBA, NuclearInstruments and Methods B 140, 1998, 402-408.

[15] Alonso U, Missana T, García-Gutiérrez M, Patelli A, Siitari-Kauppi M, Rigato V, Diffusion coefficient measurements in consolidated clay by RBS micro-scale profiling, Applied Clay Science 43, 2009, 477484.

[16] Ross GJ, Barradas NP, Hil MPl, Jeynes C, Morrissey P, Watts JF, RBS and computer simulation for the in-depth analysis of chemically modified poly(vinylidene fluoride), Journal of.Materials.Science 36, 2001, 4731-4738.

[17] Jeynes C, Barradas NP, Marriott PK, Boudreault G, Jenkin M, Wendler E, Webb RP, Elemental thin film depth profiles by IBA using simulated annealing - a new tool, Journal of Physics D Applied Physics 36, 2003, R97-R126.

[18] Rennie J, Elliott SR, Jeynes C, Rutherford backscattering study of the photodissolution of Ag in amorphous $\mathrm{GeSe}_{2}$, AppliedPhysicsLetters 48, 1986, 1430-1432.

[19] Demeulemeester J, Smeets D, Barradas NP, Vieira A, Comrie CM, Temst K, Vantomme A, Artificial neural networks for instantaneous analysis of real-time RBS spectra, NuclearInstruments and Methods B 268, 2010, 1676-1681.

[20] Barradas NP, Vieira A, Patricio R, RBS without humans, Nuclear Instruments and Methods B 190, 2002 , 231-236.

[21] Barradas NP, Vieira A, Patricio R, Artificial neural networks for automation of Rutherford backscattering spectroscopy experiments and data analysis, Physical ReviewE 65(6), 2002, 066703.

[22] Jeynes C, Barradas NP, Rafla-Yuan H, Hichwa BP, Close R, Accurate depth profiling of complex optical coatings, Surface Interface Analysis 30, 2000, 237-242.

[23] Butler JW, Criteria for validity of Rutherford scatter analysis, Nuclear Instruments and Methods B 45 , 1990, 160-165.

[24] Kirkpatrick S, Gelatt CD Jr, Vecchi MP, Optimization by simulated annealing, Science 220, 1983, 671680 . 
[25] Metropolis N, Rosenbluth AW, Rosenbluth MN, Teller AH, Teller E, Equation of state calculations by fast computing machines, Journal of Chemical Physics 21, 1953, 1087-92.

[26] Barradas NP, Jeynes C, Webb RP, Simulated annealing analysis of RBS data, Applied Physics Letters 71, 1997, 291.

[27] Barradas NP, Jeynes C, Jenkin M, Marriott PK, Bayesian error analysis of Rutherford backscattering spectra, Thin Solid Films 343-344, 1999, 31-4.

[28] Leong D, Harry M, Reeson KJ, Homewood KP, A silicon/iron-disilicide light-emitting diode operating at a wavelength of $1.5 \mu \mathrm{m}$, Nature 387, 1997, 686-688.

[29] Szilágyi E, Pászti F, Amsel G, Theoretical approximations for depth resolution calculations in IBA methods, Nuclear Instruments and Methods B 100, 1995, 103-21.

[30] Pascual-Izarra C, Barradas NP, Reis MA, Jeynes C, Menu M, Lavedrine B, Ezrati JJ, Röhrs S, Towards truly simultaneous PIXE and RBS analysis of layered objects in cultural heritage, Nuclear Instruments and Methods B 261, 2007, 426-429.

[31] Gurbich AF, Evaluated differential cross-sections for IBA, Nuclear Instruments and Methods B 268, 2010, 1703-1710.

[32] Tesmer JR, Nastasi M (Eds), 1995 Handbook of Modern Ion Beam Analysis (Pittsburgh: Materials Research Society); Wang YQ, Nastasi M (Eds), 2010 Handbook of Modern Ion Beam Analysis $\left(2^{\text {nd }}\right.$ Edition, Pittsburgh: Materials Research Society).

[33] Gurbich AF, On the concept of an actual Coulomb barrier, Nuclear Instruments and Methods B 217, 2004, 183.

[34] Bailey MJ, Howard KT, Kirkby KJ, Jeynes C, Characterisation of inhomogeneous inclusions in Darwin glass using ion beam analysis, Nuclear Instruments and Methods B 267, 2009, 2219-2224.

[35] Stoquert JP, Szörenyi T, Determination of the number and size of inhomogeneities in thin films by ion beam analysis, Physical Review B 66, 2002, 144108.

[36] Tosaki M, Energy-loss straggling caused by the inhomogeneity of target material, Journal of Applied Physics 99(3), 2006, 034905.

[37] Satoh T, Oikawa M, Kamiya T, Three-dimensional measurement of elemental distribution in minute samples by combination of in-air micro-PIXE and STIM, Nuclear Instruments and Methods B 267, 2009, 2125-2127.

[38] Ryan CG, PIXE and the nuclear microprobe: tools for quantitative imaging of complex natural materials, Nuclear Instruments and Methods B, 269 (2011) 2151-2162 


\title{
THIN FILM DEPTH PROFILING BY MEV ION BEAM ANALYSIS
}

\author{
Chris Jeynes, University of Surrey Ion Beam Centre, Guildford, England
}

\section{Problems}

\section{Problem 1}

a) Considering Figure1, estimate the sensitivity to $\mathrm{S}$.

b) Is the nice match between RBS and the chemical analysis for $\mathrm{S}$ in Table 1 any more than wishful thinking?

c) Comment on the rest of Table 1. What contribution could PIXE make in this analysis?

d) Considering Figure 2, (i) explain the high concentration of Eu at the surface. (ii) Explain the background signal above channel 750; that is, in the (black) spectrum of the unexposed material. (iii) Explain the rise of the clay signal (below channel 730) as the Eu exposure increases. (iv) How could the precision of these data be increased?

\section{Problem 2}

a) In Table 2, the precision of the capping Au layer thickness measurement is about $1 / 2 \%$. What is the absolute accuracy of this measurement? Comment on the 50\% discrepancy of the simulated annealing (columns 5, 6) and the MCMC (columns 7, 8, 9) results for this layer.

b) In Table 2, the precision of the last zirconia layer thickness measurement is about 3\%. What is its accuracy?

c) In Table 2, is it likely that silica contaminates the zirconia and vice versa (see column 3)? Comment.

\section{Problem 3}

a) In the example of Figure 10, why use a tilted incidence beam? Why use $1.13 \mathrm{MeV}$ ?

b) In Figure 10, why use two detectors? Where does (or could) information about the Ar content come from? Comment on the Ar distribution claimed.

c) In Figure 10, the depth profiles are (i) expressed in nm, and (ii) expressed in terms of layers with well-defined interfaces. Is this representation of the result required by the IBA data? What assumptions (if any) are implied? Comment. 


\section{Answers to Problems}

\section{Problem 1}

a) The noise at the $\mathrm{S}$ edge, $\sim \mathrm{ch} .605$, is about $5 \%$, that is, a signal there of about 250 counts per channel. Then the $\mathrm{K}+\mathrm{Cl}+\mathrm{Fe}$ is about 5.6 at $\%$ from Table 1 . Then the signal from the nominal 0.16 at\% $\mathrm{S}$ should be something like $1.5 \%$, considering that the $\mathrm{Z}^{2}$ for $\mathrm{S}$ is about half the average $\mathrm{Z}^{2}$ for the other elements.

b) To measure a $1.5 \%$ signal we need $0.5 \%$ counting statistics, that is an order of magnitude better than we have. With a region of interest of 100 channels we would have a chance, but for the $\mathrm{S}$ signal there are only about 20 channels. So the $\mathrm{S}$ "result" claimed for the RBS in Table 1 is spurious.

c) A properly calibrated PIXE could unequivocally determine the clay composition for the part sampled by the beam. This would give a double check on the chemical analysis and point up compositional inhomogeneities. In this case it is probably unnecessary to go to this trouble.

d) (i) The clay surface will have a network of cracks left by the polishing process. The diffusion of $\mathrm{Eu}$ into this surface modified region will follow a completely different model from (Fickian) diffusion into the bulk. This surface region should be modelled separately, and this model has little information about the material, except for telling us about the polishing quality. (ii) According to Table 1 there is $\mathrm{Sr}$ and $\mathrm{Ba}$ at low concentrations in the clay. These heavy elements will give a minimum sensitivity in the diffusion studies. (iii) The $\mathrm{Eu}$ diffusion profile goes deep, and the diffusion tail overlaps with the lighter element signals from the surface. (iv) Using a higher beam energy would look deeper into the sample.

\section{Problem 2}

a) The layer thicknesses measurements are essentially atom counting exercises. It is the signal area that is being interpreted, not the signal width (i.e. the energy loss). Of course, even at the surface the true depth resolution for this RBS is something like $10 \mathrm{~nm}$, and this means that we cannot determine the position of the interface between the Au and the first zirconia layer from the data. This is why they look mixed (see column 3)! The accuracy of counting atoms depends on the accuracy with which the charge.solid-angle product is known; this is unlikely to be better than $3 \%$ unless extraordinary care is taken. Probably, the simulated annealing measurement of the Au thickness in the first layer has included some zirconia too.

b) Again, this is an atom counting exercise, so the uncertainty is given by the absolute accuracy as above $(\sim 3 \%)$ compounded with the counting statistics uncertainty. The number of counts in the last $\mathrm{Zr}$ layer is around 5000 giving a counting statistics uncertainty of about $1.5 \%$. Thus, the absolute accuracy of the determination of the last layer thickness (note that this is an equivalent thickness from the observed number of $\mathrm{Zr}$ atoms) is essentially the same as that of the first $(\mathrm{Au})$ layer!

c) No. Zirconia and silica do not interdiffuse, and the optical properties depend on the integrity of the layers. This effect is believed to be due to a number of secondary effects possibly including roughness and probably including spectral misfitting because of incorrect stopping powers. The moral of the story is that even though an essentially perfect fit is possible, and even though apparently unequivocal and extraordinarily precise layer thicknesses are obtained from the analysis, still nagging fundamental questions remain, and incredible results are presented to the user. A modern analysis of these data would use a number of methods to yield credible results, including double scattering, stopping powers adjusted for oxides, and interface roughness. 


\section{Problem 3}

a) Tilting increases the pathlength through the thin film and therefore increases the observed depth resolution without changing the actual energy resolution of the particle detector system. For one of the detectors (the one in the IBM geometry) the $45^{\circ}$ tilt implies a $75^{\circ}$ exit angle. This nearly glancing-exit geometry gives a greatly enhanced depth resolution without greatly increasing the footprint of the beam on the sample (glancing-incidence geometry requires large and very flat samples). The use of a $\sim 1 \mathrm{MeV}$ beam makes use of the maximum of the stopping power, thus also increasing the depth resolution.

b) Two detectors used simultaneously give a "binocular" view of the sample. Occasionally, something goes wrong with one or other of the detection channels, so for every analysis it is a valuable analytical check on system integrity to have this binocular view. At tilting incidence the two detectors have quite different depth resolutions since one has a $75^{\circ}$ beam exit angle with the normal and the other has a $\sim 45^{\circ}$ beam exit angle. This is very clear in Figure 10 with the one spectrum extending over three times the energy loss of the other. The Ar signal can be seen in the tail of the low resolution signals. It can also be seen in PIXE signals, but the PIXE detector must be calibrated for absolute accuracy. The Ar is inferred to be in the Si-rich parts of the profile and not the Fe-rich parts since a high Ar content in the $\mathrm{Fe}$ signal would reduce the Fe-signal height - inconsistently with the data. The substantial Ar content is determined from its energy loss, an important part of the RBS data. PIXE data would allow us to constrain the total Ar present, but the absorption must be calculated correctly!

c) (i) The natural units of depth in IBA are $\mathrm{eV} /\left(10^{15}\right.$ atoms $\left./ \mathrm{cm}^{2}\right)$ since these are the units of the energy loss tables (this is because thin film thicknesses are most accurately determined as a mass per unit area). Therefore, to express a depth in linear terms (i.e. $\mathrm{nm}$ ) we need a density. If the $\mathrm{Fe} \& \mathrm{Si}$ profiles are expressed in terms of the disilicide (as in Figure 10), whose bulk density is known, a reasonable thickness in $\mathrm{nm}$ can be given. Thus, we impose on the data an interpretation in terms of the chemistry of the layers. This is consistent with the data, but not required by them.

c) (ii) To fit the data, not only the number of $\mathrm{Fe}$ atoms $/ \mathrm{cm}^{2}$ as a function of depth needs to be determined, but also the straggling as a function of depth. The (relatively) simple profiles shown in Figure 10 do not fit the data as they stand. To fit the data, some layer thickness nonuniformity was assumed. This is indistinguishable from fuzzy interfaces, or inter-layer diffusion. Thus, these data can be fitted equally well on a number of different physical models, and we have chosen the model which is simplest to represent: that is, assuming sharp interfaces and some non-uniformity (the details of which are not shown here). If it is necessary to distinguish between the various valid models consistent with the IBA data then other complementary techniques must be used, such as TEM or XPS. 